\title{
Computational methods for pigmented skin lesion classification in images: review and future trends
}

\author{
Roberta B. Oliveira ${ }^{1} \cdot$ João P. Papa $^{2} \cdot$ Aledir S. Pereira $^{3} \cdot$ João Manuel R. S. Tavares $^{1}$ (I)
}

Received: 30 March 2016/ Accepted: 7 July 2016/Published online: 15 July 2016

(C) The Natural Computing Applications Forum 2016

\begin{abstract}
Skin cancer is considered as one of the most common types of cancer in several countries, and its incidence rate has increased in recent years. Melanoma cases have caused an increasing number of deaths worldwide, since this type of skin cancer is the most aggressive compared to other types. Computational methods have been developed to assist dermatologists in early diagnosis of skin cancer. An overview of the main and current computational methods that have been proposed for pattern analysis and pigmented skin lesion classification is addressed in this review. In addition, a discussion about the application of such methods, as well as future trends, is also provided. Several methods for feature extraction from both macroscopic and dermoscopic images and models for feature selection are introduced and discussed.
\end{abstract}

João Manuel R. S. Tavares

tavares@fe.up.pt

Roberta B. Oliveira

roberta.oliveira@fe.up.pt

João P. Papa

papa@fc.unesp.br

Aledir S. Pereira

aledir@ibilce.unesp.br

1 Instituto de Ciência e Inovação em Engenharia Mecânica e Engenharia Industrial, Departamento de Engenharia Mecânica, Faculdade de Engenharia, Universidade do Porto, rua Dr. Roberto Frias, 4200-465 Porto, Portugal

2 Departamento de Computação, Faculdade de Ciências, Universidade Estadual Paulista, Av. Eng. Luiz Edmundo Carrijo Coube, 14-01, Bauru, SP 17033-360, Brazil

3 Departamento de Ciências de Computação e Estatística, Instituto de Biociências, Letras e Ciências Exatas, Universidade Estadual Paulista, rua Cristóvão Colombo, 2265, São José do Rio Preto, SP 15054-000, Brazil
Furthermore, classification algorithms and evaluation procedures are described, and performance results for lesion classification and pattern analysis are given.

Keywords Pattern analysis - Feature extraction and selection - Classification methods · Macroscopic and dermoscopic images

\section{Introduction}

Computational methods for skin cancer diagnosis have been proposed in order to aid dermatologists in early assessment of skin cancer and in the follow-up of pigmented skin lesions [1-3]. Such lesions represent an abnormal production of melanocytes cells, which are mainly caused by excessive sun exposure. Melanocytes cells are responsible for creating the substance melanin, one of the functions of which is to provide pigmentation in the skin. Furthermore, the number of skin cancer cases has increased in the last years, and consequently, an increasing number of deaths caused by this disease has been reported, particularly due to melanoma cases (Fig. 1c, d) [4-6]. Therefore, pigmented skin lesions have been a cause for global concern, since some types of benign lesions may become skin cancer, such as dysplastic nevi (Fig, 1a, b).

Image acquisition, pre-processing, segmentation, feature extraction, and classification are fundamental steps commonly found in computational systems for diagnosing skin lesions. Different non-invasive imaging techniques have been used to assist dermatologists [8]. Macroscopic images $[9,10]$ and dermoscopic images [11, 12] are examples of images acquired from such techniques that have been widely used in the diagnosis of pigmented skin lesions by computational methods. Macroscopic images (Fig. 1a, c), 

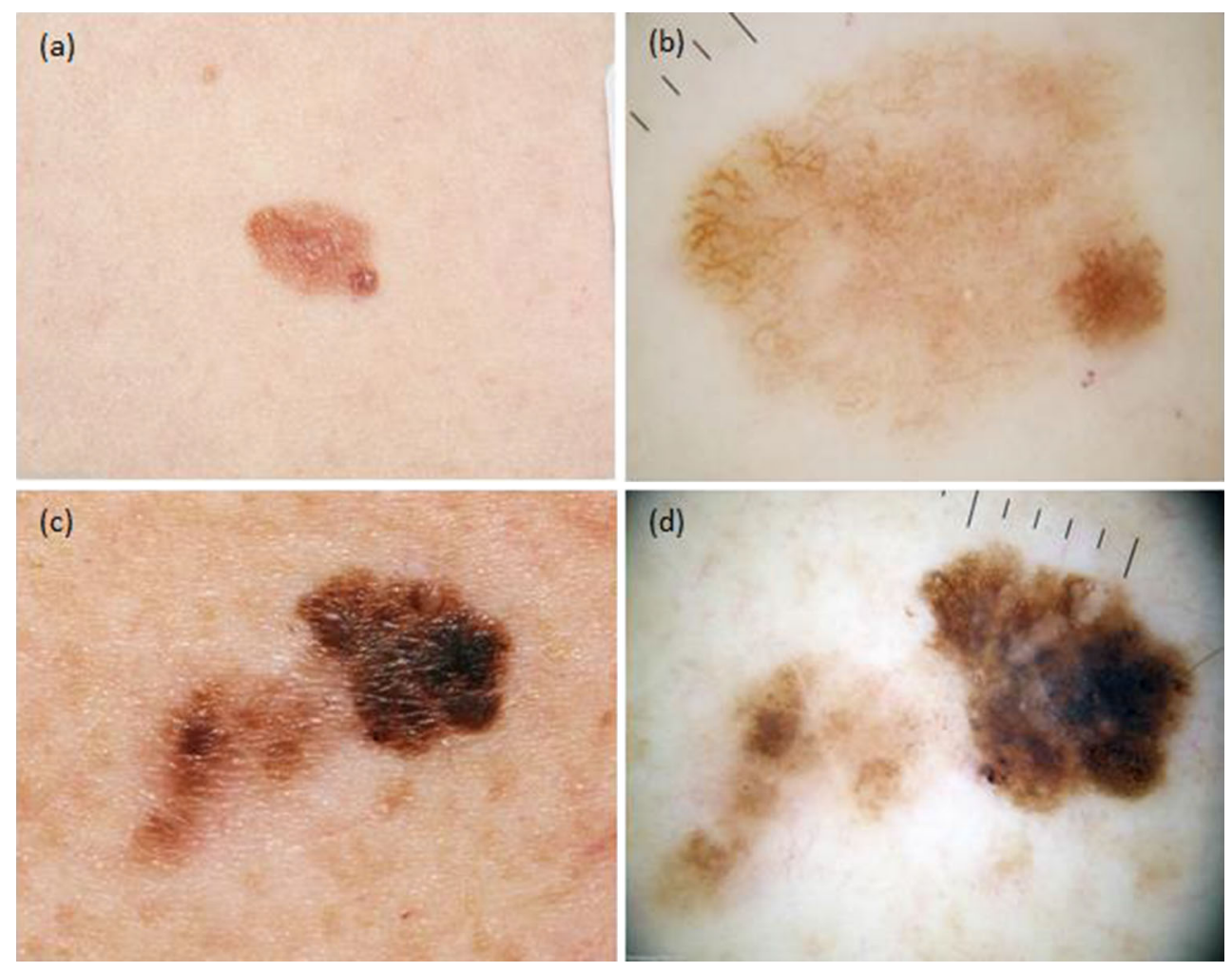

Fig. 1 Two examples of macroscopic images $(\mathbf{a}, \mathbf{c})$ and dermoscopic images $(\mathbf{b}, \mathbf{d}): \mathbf{a}, \mathbf{b}$ are images of a dysplastic nevus and, $\mathbf{c}, \mathbf{d}$ are of an invasive melanoma (images publicly available from Bourne et al. [7])

commonly known as clinical images, are usually acquired from standard cameras or mobile devices. On the other hand, dermoscopic images (Fig. 1b, d) may be acquired from dermatoscope devices or specific cameras in order to better visualize the pigmentation pattern on the skin surface. However, their imaging conditions are frequently inconsistent; for example, macroscopic images can be acquired from variable distances and/or under different illumination conditions. Furthermore, the images may have poor resolution, which may be challenging when the lesion under study is small. An additional problem with both macroscopic and dermoscopic images is related to the presence of artefacts, such as hair, reflections, shadows, skin lines, and bubbles, which may hinder adequate analysis of the imaged skin lesions.

The identification of the regions of the lesions in such images may be performed in order to assist in the process of classification [13]. Segmentation is an important step that allows the extraction of such regions of interest (ROI) from an image [14-34]. However, before the segmentation step, previous pre-processing methods are usually applied to reduce the effects of undesirable artefacts that may influence the outcome of the segmentation step. These methods can be based on colour space transformation [20, 26, 35], illumination correction [36, 37], contrast enhancement [20, 22, 23, 26, 38-42], artefact removal [14, 20, 21, 43, 44], and approximate lesion localization [45]. In addition, hair removal methods are also used in pre-processing steps, since this artefact may considerably affect the detection of lesion borders [46-53]. Lee et al. [54] proposed a solution for hair removal, especially thick dark hairs, which is based on one of the first widely adopted methods for hair removal in dermoscopy images, and consists of identifying the hair location, replacing the values of the detected hair pixels in the original image by the values of the corresponding nearby non-hair pixels and smoothing the thin lines. An overview of lesion border detection methods, including the pre-processing, segmentation, and post-processing steps, is presented in Celebi et al. [55, 56]. In addition, the authors also discuss performance evaluation issues and propose guidelines for future studies.

Computational methods for pigmented skin lesion classification are usually based on the features of the pixels within the segmented ROIs. Therefore, the extraction of representative features of the ROIs under analysis is an important step for the efficient classification of the segmented lesions. In this step, common difficulties are: (1) identification of the features to be used; (2) to confirm that the number of selected features is sufficient to describe the classification problem; (3) the number of selected features is too large, which requires high computational resources; and (4) there are redundant and/or irrelevant features that 
should be removed from the feature set. Techniques to reduce the dimensionality of the data may be used to solve these problems according to one of the following reduction strategies: feature transformation (also known as feature extraction in the literature concerning pattern recognition $[57,58]$ ), and feature selection [59].

The feature extraction strategy allows the modification of all the data of the image, in order to emphasize the most effective features, ensuring the correct separation of the classification classes [57]. Such strategy is based on the generation of a new feature space, which may expand or reduce, according to the adopted strategy. The new features may be extracted by means of discovery of missing information from relationships among the features, or even by means of searching for a new feature space with smaller dimensions through functional mapping. Contrary, new features are not created in the feature selection strategy, meaning that a subset from the original features is defined when using this approach. Both strategies may also be combined in order to achieve a better representation of the features. For example, in cases in which the feature extraction step increases the number of features, feature selection algorithms can provide an automatic reduction of such excessive features. Furthermore, a larger feature space may include redundant or irrelevant data [60].

Several solutions [61-64] have been proposed for feature extraction and selection of pigmented skin lesions, in order to represent them according to a certain clinical criteria [65-67]. Such features may be used for the classification process, in order to provide dermatologists with a computer-aided diagnosis of pigmented skin lesions $[2,12]$. In this review, some of the most relevant solutions that have been developed to assist the skin lesion diagnosis from macroscopic and dermoscopic images are introduced, including those concerning the steps of feature extraction and selection, and image classification. Hence, this review is highly valuable for those wishing the design and/or implementation of competent expert systems for the automated classification of skin lesions in images.

This paper is organized as follows: a review of the main computational methods that have been applied to extract and select features from macroscopic and dermoscopic images of pigmented skin lesions is presented in Sect. 2. The main focus of that section is on the feature extraction step according to several clinical criteria. In addition, the feature selection process is addressed. The current state-of-the-art concerning the pigmented skin lesion classification, including the advantages and disadvantages of the reviewed methods, evaluation measures, and performance results for pattern and lesion classification, is presented in Sect. 3. Finally, conclusions and future trends about the computational methods of pigmented skin lesion classification are pointed out in the last section.

\section{Image analysis of pigmented skin lesions}

Computational methods regarding the feature extraction have been commonly developed based on the $\mathrm{ABCD}(\mathrm{E})$ rule, pattern analysis, seven-point checklist, and Menzies' method, which are examples of clinical approaches used for the diagnosis of skin cancer from images [67-69]. The first approach can be used to extract features from both macroscopic and dermoscopic images, whereas the other approaches are usually applied to dermoscopic images in order to identify more detailed pattern features on the surfaces of the lesions. The feature analysis based on these approaches, as well as the feature selection and extraction steps, is presented with details in the following sections.

\subsection{Feature analysis based on clinical approaches}

The $\mathrm{ABCD}(\mathrm{E})$ rule is based on asymmetry, border, colour, diameter (or differential structures in the case of dermoscopic images), and evolution (or elevation) features, according to the criteria presented in Table 1 . Such rule has been widely used for the feature extraction and automatic diagnosis of pigmented skin lesions [10, 70].

The feature extraction based on pattern analysis has also been used for the pigmented skin lesion automatic diagnosis [71-74]. This approach assists in diagnosis by determining the presence of specific patterns visible in dermoscopic images, which may be divided into global and local patterns [75], as detailed in Table 2. Global patterns are represented by textured structures present in most of the lesions. Some examples of such patterns are illustrated in Fig. 2. Local patterns are dermoscopic structures. Such patterns may be present or absent, as well as presenting irregular/regular or atypical/typical structures, as indicated in Table 2, which may define the type of lesion or whether it is benign or malignant. Examples of such patterns are illustrated in Fig. 3.

The pattern analysis consists of examining the size, uniformity, and distribution of the above-mentioned patterns. The benign lesion structures are usually uniform; in other words, the lesions do not present several patterns in their structure. Therefore, the presence of at least three (multicomponent), parallel or non-specific global patterns indicates a higher probability of being a melanoma (malignant lesion). Furthermore, the presence of local patterns, such as blue-whitish veil and regression structures, or even some patterns considered atypical, irregular, or asymmetric may identify a melanoma [75]. Due to the low number of criteria to be analysed, the seven-point checklist and Menzies' method were introduced for skin lesion diagnosis from dermoscopic images in order to simplify the common pattern analysis [67]. The criteria of both clinical approaches are detailed in Table 3. 
Table 1 Criteria of the $\mathrm{ABCD}(\mathrm{E})$ rule for the diagnosis of skin cancer from clinical and dermoscopy analysis

\begin{tabular}{|c|c|c|c|c|c|c|}
\hline \multirow[t]{2}{*}{ Feature } & \multicolumn{2}{|l|}{ Clinical analysis } & \multirow[t]{2}{*}{ Feature } & \multicolumn{3}{|l|}{ Dermoscopy analysis $^{\mathrm{a}}$} \\
\hline & Benign lesion & Malignant lesion & & Definition & Score & $\begin{array}{l}\text { Weight } \\
\text { factor }\end{array}$ \\
\hline Asymmetry (A) & $\begin{array}{l}\text { Shape is } \\
\text { symmetric }\end{array}$ & Shape is asymmetric & $\begin{array}{l}\text { Asymmetry } \\
\text { (A) }\end{array}$ & $\begin{array}{l}\text { Border, colours, or structures are } \\
\text { asymmetric in } 0,1 \text {, or } 2 \text { perpendicular } \\
\text { axes }\end{array}$ & $0-2$ & 1.3 \\
\hline Border (B) & $\begin{array}{r}\text { Border is regular } \\
\text { or well defined }\end{array}$ & $\begin{array}{l}\text { Border is irregular or ill- } \\
\text { defined }\end{array}$ & Border (B) & $\begin{array}{l}\text { Abrupt cut-off of network at the border } \\
\text { in } 0-8 \text { segments }\end{array}$ & $0-8$ & 0.1 \\
\hline Colour (C) & $\begin{array}{l}\text { Colours are } \\
\text { uniform }\end{array}$ & Colours are non-uniform & Colour $(\mathrm{C})$ & Presence of six possible basic colours ${ }^{b}$ & $1-6$ & 0.5 \\
\hline Diameter (D) & Size $<6 \mathrm{~mm}$ & Size $\geq 6 \mathrm{~mm}$ & $\begin{array}{l}\text { Differential } \\
\text { structural } \\
\text { (D) }\end{array}$ & $\begin{array}{l}\text { Presence of five differential structural } \\
\text { components }{ }^{c}\end{array}$ & $1-5$ & 0.5 \\
\hline Evolution (E) & No change & $\begin{array}{l}\text { Changes in size, shape, or } \\
\text { shades of colour features }\end{array}$ & & & & \\
\hline Elevation (E) & Smooth surface & High surface & & & & \\
\hline \multicolumn{7}{|c|}{$\begin{array}{l}\text { Total dermatoscopy score }(\text { TDS })=(\text { A score } \times 1.3)+(\text { B score } \times 0.1)+(\mathrm{C} \text { score } \times 0.5)+(\mathrm{D} \text { score } \times 0.5) \text {. Diagnosis: TDS }<4.75 \text {, benign } \\
\text { melanocytic lesion; TDS of } 4.75-5.45 \text {, suspicious lesion; TDS }>5.45 \text {, lesion highly suspicious for melanoma }\end{array}$} \\
\hline \multicolumn{7}{|c|}{ b White, red, light brown, dark brown, blue-grey, and black } \\
\hline \multicolumn{7}{|c|}{ c Network, structureless areas, branched streaks, dots, and globules } \\
\hline \multirow{9}{*}{$\begin{array}{l}\text { Table } 2 \text { Pattern } \\
\text { dermoscopic ima }\end{array}$} & $\begin{array}{l}\text { analysis in } \\
\text { ges }\end{array}$ & \multicolumn{2}{|l|}{ Global pattern } & \multicolumn{3}{|l|}{ Local pattern } \\
\hline & & \multicolumn{2}{|l|}{ Reticular } & \multicolumn{3}{|c|}{$\begin{array}{l}\text { Pigmented network (present or absent/typical or } \\
\text { atypical) }\end{array}$} \\
\hline & & \multicolumn{2}{|l|}{ Globular } & \multicolumn{3}{|c|}{ Dots/globules (present or absent/regular or irregular) } \\
\hline & & \multicolumn{2}{|l|}{ Cobblestone } & \multicolumn{3}{|c|}{ Streaks (present or absent/regular or irregular) } \\
\hline & & \multicolumn{2}{|l|}{ Homogeneous } & \multicolumn{2}{|l|}{ Blue-whitish veil (present or absent) } & \\
\hline & & \multicolumn{2}{|l|}{ Starburst } & \multicolumn{3}{|c|}{$\begin{array}{l}\text { Blotches or pigmentation (present or absent/regular } \\
\text { or irregular) }\end{array}$} \\
\hline & & \multicolumn{2}{|l|}{ Parallel } & \multicolumn{3}{|l|}{ Hypopigmentation (present or absent) } \\
\hline & & \multicolumn{2}{|c|}{$\begin{array}{l}\text { Multicomponent (combination of three or more } \\
\text { global patterns) }\end{array}$} & \multicolumn{3}{|c|}{ Regression structures (present or absent) } \\
\hline & & \multicolumn{2}{|l|}{ Non-specific (absent patterns) } & \multicolumn{3}{|l|}{ Vascular structures (present or absent) } \\
\hline
\end{tabular}

The seven-point checklist has been applied in the literature to achieve better accuracy for the computational diagnosis of dermoscopic images [24, 78, 79]. This method consists basically of seven criteria based on local patterns that may be applied to diagnose the malignancy in pigmented skin lesions, particularly melanomas, which are divided into major and minor criteria [75]. A total score of three or more points is more likely to be melanoma, for which the presence of each major criterion receives two points and each minor criterion receives one point [67]. The Menzies' method allows for identifying colour patterns within the lesion and the asymmetry along any axis drawn through the centre of the lesion, as well as the number of positive features [67]. In malignant lesions, particularly melanomas, an asymmetric pattern, more than one colour, and at least one positive feature are usually presented, whereas the benign lesions present a symmetric pattern and only one colour [67]. Computational methods based on the Menzies' criteria have been proposed to analyse the presence of six basic colour classes (white, red, light brown, dark brown, blue-grey, and black) for dermoscopic images $[80,81]$.

\subsection{Feature extraction}

Skin lesion features can be extracted either according to a global or local manner in order to obtain information for classification. The most of works explore the global features of the lesion, i.e. extract features from all segmented region [82]. However, some studies have used local features, which allow the characterization of different region 
Fig. 2 Examples of global patterns in dermatoscopy images: a reticular, b globular, c cobblestone, $\mathbf{d}$ homogeneous, e parallel, and $\mathbf{f}$ starburst (images available in Argenziano et al. [76])
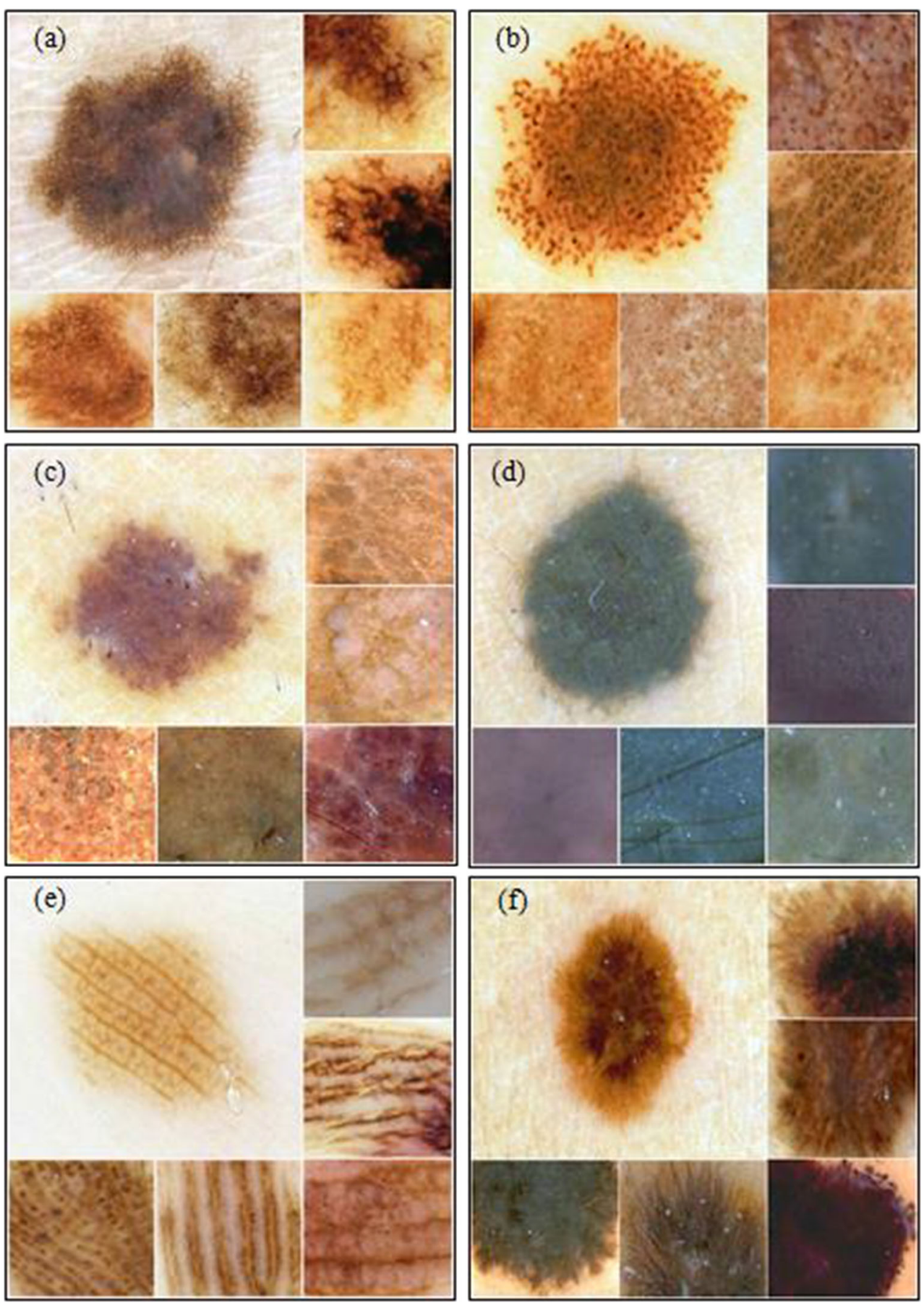

of the lesion. Bag-of-feature $(\mathrm{BoF})$ approach is a simple strategy that has been used to compute local features [11, 71, 83-85]. In general, skin lesion features are categorized into shape features, colour variation and/or texture analysis $[9,86]$. These features can be extracted to detect patterns [73] or diagnose skin lesions [82] from both macroscopic and dermoscopic images. Extracted features of pigmented skin lesions from both of images are summarized in Table 4 and discussed in the following sections.

\subsubsection{Shape features}

Shape features allow the assessment of lesion's asymmetry or border's irregularity. The asymmetry features may be examined according to dividing the region of the lesion under analysis into two sub-regions by an axis of symmetry, in order to analyse the similarity of the area by overlapping the two sub-regions of the lesion along the axis. From such an axis, the asymmetry index may be calculated by the difference between the two sub-regions of 


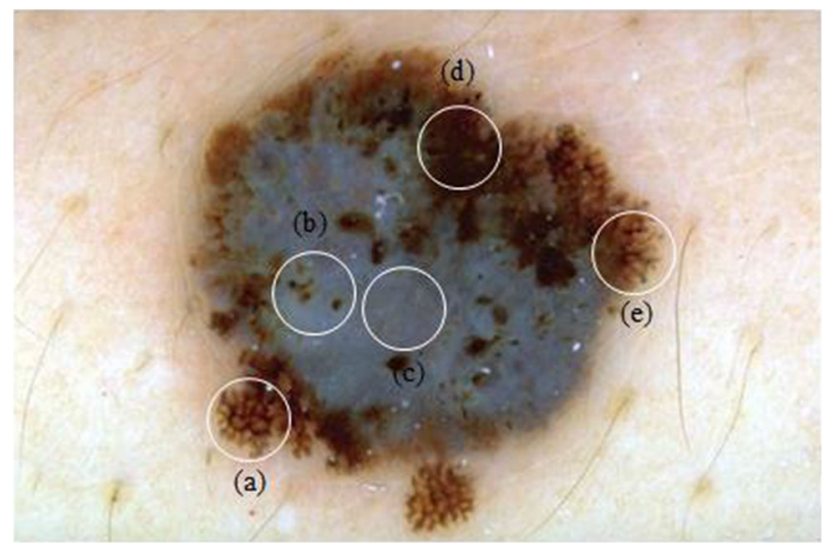

Fig. 3 Examples of local patterns in a dermatoscopy image: a atypical pigmented network, b irregular dots/globules, c blue-whitish veil, $\mathbf{d}$ irregular pigmentation and $\mathbf{e}$ irregular streaks (adapted from Celebi et al. [77]) (colour figure online)

the lesion; for example, by applying the XOR operation between them [87]. In some studies, the axis of symmetry is defined based on the principal axis of inertia [87], major and minor axis orientation [12,88] and longest or shortest diameter [89].

Geometrical measures from the segmented lesion area have been commonly computed for assessing the lesion's asymmetry and border's irregularity [12, 63, 70, 84, 129]. Such measures include the area of the lesion (computed as the number of pixels inside the lesion region [10] or by applying the bit quads method [88]), aspect ratio, compactness, perimeter, greatest diameter, shortest diameter, equivalent, convex hull, eccentricity, solidity, rectangularity, entropy measures, circularity index (namely thinness ratio), and irregularity index. Shape features based on wavelet transform [12, 61, 106], Fourier transform [104], and fractal dimension $[92,103]$ have also been used for assessing the border's irregularity.

Shape features of differential structures inside the lesion in dermoscopic images may also be considered [66], such as solid pigments of the lesions computed according to Chang et al. [87]. In other studies [63, 93, 96], the asymmetry is assessed, according to pre-defined regions inside the lesion under analysis.

In order to identify the sharp transition between inside and outside regions of a lesion concerning its border, Iyatomi et al. [94, 96] divided the lesion region into eight equiangular regions. For each region, the ratio of the colour intensity inside and outside the lesion and the gradient of the colour intensity were computed in particular colour channels, according to a pre-defined window centred at the border of the lesion, whereas, Celebi et al. [88] computed the differences and ratios of two statistics (mean and standard deviation) over a particular colour channel, considering the following regions: lesion and inner and outer peripheral regions relative to the border of the lesion.

\subsubsection{Colour variation}

The RGB colour space is commonly used to represent the colours of skin lesions $[63,110]$. Other colour spaces have also been applied in order to obtain more specific information about a lesion's colours, such as: normalized RGB [110, 111], HSV [11, 84, 110], HVC [109], CMY [108], YUV [108], I1/2/3 [110], Opp [83, 84], $\bar{I}_{i}^{N}$ [70], JCh [73], $L * C * H$ [87], CIEXYZ [111], CIELAB [11, 83, 84], and CIELUV $[11,110]$.

Table 3 Diagnostic criteria included the seven-point checklist and Menzies' method

\begin{tabular}{|c|c|c|c|c|}
\hline \multicolumn{2}{|l|}{ Seven-point checklist ${ }^{\mathrm{a}}$} & \multicolumn{3}{|l|}{ Menzies' method $^{\mathrm{d}}$} \\
\hline Major criteria $^{\mathrm{b}}$ & Minor criteria ${ }^{c}$ & Colour of lesion & Symmetry of pattern & Positive feature \\
\hline $\begin{array}{l}\text { Atypical pigmented network } \\
\text { Blue-whitish veil } \\
\text { Atypical vascular pattern }\end{array}$ & $\begin{array}{l}\text { Irregular streaks } \\
\text { Irregular pigmentation } \\
\text { Irregular dots/globules } \\
\text { Regression structures }\end{array}$ & $\begin{array}{l}\text { One colour } \\
\text { More than one colour }\end{array}$ & $\begin{array}{l}\text { Symmetrical pattern } \\
\text { Asymmetrical pattern }\end{array}$ & $\begin{array}{l}\text { Blue-whitish veil } \\
\text { Multiple brown dots } \\
\text { Pseudopods } \\
\text { Radial streaming } \\
\text { Scar-like depigmentation } \\
\text { Peripheral black dots/globules } \\
\text { Multiple colours (5 or 6) } \\
\text { Multiple blue/grey dots } \\
\text { Broad pigment network }\end{array}$ \\
\hline
\end{tabular}

\footnotetext{
${ }^{\text {a }}$ Seven-point total score $<3=$ non-melanoma or $\geq 3=$ melanoma

b Major criteria receive two points

${ }^{c}$ Minor criteria receive one point

${ }^{\mathrm{d}}$ Diagnosis for benign lesions (symmetrical pattern and one colour) and malignant lesions (asymmetrical pattern, more than one colour and at least one positive feature)
} 
Table 4 Extracted features of pigmented skin lesions from both macroscopic and dermoscopic images

\begin{tabular}{|c|c|}
\hline Feature & References \\
\hline \multicolumn{2}{|l|}{ Shape } \\
\hline Asymmetry index & {$[87,90]^{\mathrm{a}} ;[12,84,88,91]^{\mathrm{b}}$} \\
\hline Statistical geometrical measures & {$[9,10,61,87,92]^{\mathrm{a}} ;[12,63,84,88,89,93-103]^{\mathrm{b}}$} \\
\hline $\begin{array}{l}\text { Statistical measures based on border's gradient or periphery } \\
\text { regions }\end{array}$ & {$[9,10,61,70,104]^{\mathrm{a}} ;[74,78,84,88,93,94,96,97,105]^{\mathrm{b}}$} \\
\hline Border features (irregularity index) & {$[61,90,92,104]^{\mathrm{a}} ;[12,89,103,106,107]^{\mathrm{b}}$} \\
\hline \multicolumn{2}{|l|}{ Colour } \\
\hline Statistical measures based on colour models & {$[9,10,70,82,87,90]^{\mathrm{a}} ;[63,71,73,84,88,93,94,96,101,105,108-113]^{\mathrm{b}}$} \\
\hline Colour occurrence or percentage & {$[74,81,102,103,113-116]^{\mathrm{b}}$} \\
\hline Absolute or relative colour features & {$[87]^{\mathrm{a}} ;[77,88,93,94,96,99,113,117-119]^{\mathrm{b}}$} \\
\hline Colour asymmetry & {$[73,88,105,110]^{\mathrm{b}}$} \\
\hline Histogram-based features (colour distribution) & {$[24,62,78,79,83,84,88,97,99,102,110,112]^{\mathrm{b}}$} \\
\hline Colour features based on cluster analysis & {$[102,112,120]^{\mathrm{b}}$} \\
\hline Border's gradient-based features & {$[96,113]^{\mathrm{b}}$} \\
\hline \multicolumn{2}{|l|}{ Texture } \\
\hline Statistical & $\begin{array}{l}{[9,10,70]^{\mathrm{a}} ;} \\
\quad[11,63,64,77,83,84,88,93,94,96,101,102,105,108-112,120-122]^{\mathrm{b}}\end{array}$ \\
\hline Model based & {$[90]^{\mathrm{a}} ;[12,123,124]^{\mathrm{b}}$} \\
\hline Filter based & {$[12,24,62,73,78,79,83,84,111,112,121,123]^{\mathrm{b}}$} \\
\hline \multicolumn{2}{|l|}{ Other features } \\
\hline Colour-texture features & {$[82]^{\mathrm{a}} ;[72,124]^{\mathrm{b}}$} \\
\hline High-level intuitive features & {$[125]^{\mathrm{a}}$} \\
\hline Manual information & {$[10,82]^{\mathrm{a}}$} \\
\hline Diameter & {$[102]^{\mathrm{b}}$} \\
\hline Differential structures & {$[103,126]^{\mathrm{b}}$} \\
\hline Evolution measures & {$[127]^{\mathrm{a}} ;[128]^{\mathrm{b}}$} \\
\hline
\end{tabular}

${ }^{\text {a }}$ Macroscopic images

b Dermoscopic images

Statistical measures are widely applied to the feature extraction from skin lesion images [10, 63, 70, 93]. The minimum, maximum, average, standard deviation, skewness, and variance are examples of such measures, which may be computed for each colour channel of the lesion region by using one or several colour models. Furthermore, these measures may also be applied to other regions associated with the lesion's border, in order to identify a sharp transition between them, which indicates malignancy. The background skin (normal skin), and surrounding skin (inner or outer peripheral regions) are examples of such regions, which may be considered as part of the lesion. Peripheral regions may be defined by a recursive erosion process [93, 110], a fast Euclidean distance transform algorithm [88], or a circular region with centre point upon the lesion's centroid [87]. In addition, such regions may reduce the effects of peripheral inflammation and errors caused by automatic border detection, as proposed by Celebi et al. [88].

Skin lesion features based on relative colours have been proposed [77, 88, 93], in order to assess colour features from the different regions associated with the lesion. The relative colour consists of comparing each pixel value of the lesion to the average colour value of the surrounding skin. Furthermore, this feature may present advantages such as compensating the variation of colour of the image caused by illumination and equalizing variations in skin colour among individuals [77].

The occurrence of the possible basic colours present in the skin lesions has also been analysed [74, 81], as well as the number or percentage of pixels within the segmented area for each of the basic colours $[9,73]$.

\subsubsection{Texture analysis}

Texture analysis is frequently considered for image analysis of skin lesions, since it assists in discriminating between benign and malignant lesions by measuring the roughness of their structure. Texture descriptors with statistical-, model-, and filter-based approaches [130] have been used for texture quantification of skin lesions. Among 
the various statistical-based texture descriptors applied in the literature, the grey-level co-occurrence matrix (GLCM) proposed by Haralick et al. [131] has been one of the most commonly used $[63,84,101,105,110,111]$. The GLCM is a statistical measure that computes the joint probability of occurrence of grey-levels considering two pixels spatially separated by a fixed vector. Several measures may be computed based on the GLCM, such as variance, entropy, dissimilarity, correlation, contrast, energy, maximum probability, inverse difference, angular second moment (ASM), mean, standard deviation, and homogeneity. In Schaefer et al. [110], the authors computed the ratio and difference of the same co-occurrence features between different image regions.

Skin lesion features from histograms, which are also statistical-based descriptors, are extracted by some researchers to represent texture features [11, 84]. Tanaka et al. [121] computed some aforementioned statistical measures based on the intensity histogram, whereas Barata et al. [83] applied gradient histograms, such as the gradient amplitude and orientation to represent the texture feature. In order to compute the image gradient, the authors applied a Gaussian filter to the grey-level image for further computation of the gradient vector at each pixel using the wellknown Sobel filter. Local binary pattern (LBP) that is a discriminative rotation invariant feature descriptor [84, 102, 112], statistical measures based on pixel intensities [9, 70], run-length matrix [121], and entropy features [120], has also been applied to texture extraction based on statistical approaches.

Model-based texture descriptors have also been proposed to assess the skin lesion's texture, such as fractal dimensional [12], auto-regression [123], and Markov random fields (MRF) [124]. Among these, fractal dimension has been applied with the box-counting method (BCM), being one of the most commonly used methods, since it is simple and effective [132]. Image-based fractal dimension [132] is a procedure for splitting the image in several quadrants to quantify the irregularity level or self-similarity of the image's fractals.

Wavelet transform [12, 71, 112], Fourier transform [24, 78, 79, 112, 121], Gabor filtering [83, 84, 123], scaleinvariant feature transform (SIFT) [84], and steerable pyramid transforms [73], which are filter-based texture descriptors, have also been proposed for feature extraction of skin lesion images. Such descriptors allow the decomposition of the input image into component parts in order to extract features from the structures of interest. Sobel, Hessian, Gaussian, and difference of Gaussians (DoG) features have also been extracted based on bank of Gaussian filters [111]. Further details regarding texture analysis techniques for image feature extraction are presented in Xie [130].

\subsubsection{Other features}

Skin lesion features based on shape, colour, and texture properties have been commonly used for skin lesion recognition. However, other features have also been considered, such as information regarding the part of the body, size, and gender, since they can assist in skin lesion diagnosis $[10,82]$. Colour-texture descriptors have also been recently used to assess skin lesion features, e.g. colour image analysis learning vector quantization (CIA-LVQ) in the RGB colour space [82] and joint distribution of colour (JDC) in the $L^{*} a^{*} b^{*}$ colour space [72]. Further details regarding colour-texture descriptors are presented in Xie [130].

The lesion's diameter is another feature that can be used for skin lesion diagnosis. This feature is examined according to the size of the lesion, which is defined by the greatest distance between any two points of a lesion's edge [65]. This feature is not commonly applied to skin lesion classification due to its great dependence on the image resolution [88], since the image size affects the number of pixels for each segmented lesion's region. An application of this feature is presented in Møllersen et al. [102], in which the diameter of a lesion is defined as the length of the major axis of the best-fit ellipse. The differential structures of skin lesions may also be assessed, more specifically in dermoscopic images. For example, in Torre et al. [126], multidimensional receptive field histograms (MFHs) were obtained by means of Gaussian derivatives and a Laplacian Gaussian operator, in order to reproduce features of the local differential structures of skin lesions.

Elevation and evolution features can be assessed to assist in skin lesion classification process [66, 133]. The former is a morphological feature that may be measured considering its surface. The latter may represent the historical evolution of the lesion in order to diagnose it, including changes in its shape, size, shades of colour, or surface features. To the best of our knowledge, few previous image analysis systems of skin lesions surveyed in the literature have used such features [127, 128]. One of the reasons may be related to the complexity of feature extraction from the elevation criterion, or even the unavailability of a database with at least two images of the same lesion that must be taken over time to assess its evolution.

Three-dimensional digital imaging may be designed to extract information about the elevation feature of skin lesions. For example, Hani et al. [134] and Fadzil et al. [135] proposed a method to measure the thickness of some skin lesion types from the 3D surface image. Lesion's thickness is the elevation present between the base and the surface of the lesion. In addition, registration methods may 
be applied to track skin lesions in images [136], or to detect changes in their structure over time, as the algorithm introduced by Huang and Bergstresser [127]. The authors proposed a new method for the melanoma registration, based on bipartite graph matching, in order to find sufficiently good correspondences between successive images of multiple skin lesions. The authors used the Voronoi cells and distances between points to transform the point registration problem in images to a bipartite graph matching problem.

\subsection{Feature selection}

A feature selection step [137] has been used for pattern analysis and skin lesion classification in order to select the most relevant features and reduce the dimensionality of the feature space so that irrelevant and/or redundant features are removed [93, 98, 108, 121]. Moreover, such features may influence the performance of the classification process, i.e. render it a slower process [138]. Several benefits are associated with the application of feature selection schemes, such as [88]: (1) to reduce the feature extraction time, (2) to decrease the classification complexity, (3) to improve the classification accuracy rate, (4) to decrease training and testing time, and (5) to simplify the understanding and visualizing the data.

Essentially, the feature selection process has the following steps: (1) feature subset selection, (2) feature subset evaluation, (3) stopping criterion, and (4) validation procedure [139]. Search strategies may be applied to define candidate subsets from extracted features of skin lesions, which are evaluated and compared to the previous best subset until a given stopping criterion is reached. This process is iterative, and it only finishes when it reaches the established stop criterion. Thus, the selected best subset should be verified for the specific problem, i.e. the skin lesion classification.

Feature subset selection step consists of finding features through a given process of heuristic searches in order to identify a candidate feature subset for evaluation. Several search algorithms, such as best-first [108], ranker [12, 108], incremental stepwise [93, 98] and random [87, 88], have been used for the feature subset selection process. Exhaustive and genetic searches are other examples of such algorithms that may be applied [140]. These algorithms influence the search direction and execution time of the selection process depending on the adopted search strategy, which may be complete, sequential, or random [139, 141]. Another model to establish a feature subset is applying embedded methods such as decision tree algorithms, which incorporate the feature selection in its training process [138].

In evaluation step, the selected feature subset is then evaluated according to the type of search algorithm applied before. The filter model [141] has been commonly used for the evaluation process of skin lesion feature selection. This model allows for evaluating the goodness of selected features without using any classification algorithms. Each candidate subset is evaluated by means of applying an independent criterion, which may be based on distance, ${ }^{1}$ information, ${ }^{2}$ dependency, ${ }^{3}$ or consistency ${ }^{4}$ measures, in order to compare it with the best current subset previously established. If the evaluated subset is considered the best, it becomes the best current subset. Examples of filter methods applied in the literature based on the aforementioned independent measures are: gain ratio feature selection (GRFS) [12], information gain measure [12], Chi-squared [12], correlation-based feature selection (CFS) [10, 12, 61], ReliefF [12, 88], mutual information-based feature selection (MIFS) [88], sequential feature selection (SFS) [80], generalized sequential feature selection (GSFS) [108], and fast correlation-based feature filter (FCBF) [110].

Wrapper [142], hybrid [141], and embedded [138] models can also be used to evaluate the selected feature subset by a search strategy. The evaluation of feature subsets based on the wrapper model is similar to the filter model. The main difference between these two models is the use of classification algorithms to evaluate each candidate subset in order to determine the most relevant subset, for which the classification algorithm tends to perform better when searching for such a subset [141]. The hybrid model combines properties of filter and wrapper models to evaluate feature subsets in order to consider the advantages of both models, as well as to deal with large data sets. The embedded model has a built-in mechanism to perform the feature selection; it incorporates the feature selection as part of the training process. The decision tree induction algorithms, such as classification and regression tree (CART), are examples of such a model [137].

Feature selection methods based on a filter model [141] are more often preferred to other models due to the following advantages: computationally efficient, simpler and faster methods, independent evaluation criteria, and ability to overcome over-fitting $[10,12,98]$. Nevertheless, the features selected by using a filter model may not be the most relevant for the application, whereas the wrapper model [142] may be applied to search for the most relevant features based on classification algorithms to improve the

\footnotetext{
${ }^{1}$ These measures try to find the feature that may separate the classes as far as possible by greater distance between them.

2 These measures establish the information gain from a feature.

3 These measures are also known as correlation measures applied to evaluate the ability to predict the value of one feature from the value of another.

${ }^{4}$ These measures consist of finding a minimum number of features that may separate classes as consistently as the full set of features may.
} 
performance of the feature selection. The wrapper model is not commonly applied due to the high computational time, as demonstrated by Celebi et al. [88]. However, efficient search strategies may be proposed for this model to avoid the time-consuming task of classifying skin lesions. Although the hybrid model inherits the advantages of both filter and wrapper models, this model may be complex and also inherits the disadvantages of wrapper model. Methods based on an embedded model provide simplicity and a faster solution for the feature selection step compared to methods based on the filter model [137].

The stopping criterion determines the situation in which the feature selection process must stop. Some examples of such criteria occur when: (1) the search is complete, (2) the predefined minimum number of features is achieved, (3) the predefined maximum number of the process is achieved, and (4) addition or removal of any feature occurs that worsens the outcome of the best found subset until that moment [141]. The validation procedure consists of verifying the best feature subset established by the previous steps. Hence, the validation process may be performed upon applying classifiers from a new set of features in order to measure the classification performance or error rate of the selected feature subset.

Principal component analysis (PCA) [143] and linear discriminant analysis (LDA) [62], which are methods for space dimensionality reduction, have also been applied to feature selection [24, 70, 73]. Maglogiannis and Doukas [108] applied several classification methods to evaluate the obtained subsets by using feature selection algorithms such as the CFS, PCA, and GSFS. Furthermore, the achieved results are compared to the ones obtained from all features without applying any feature selection algorithm. The authors concluded that the application of feature selection algorithms may reduce the complexity of the classification. On the other hand, the performance is not always good and is highly dependent upon the classifier. Therefore, they opted to use all features for the skin lesion classification. On the other hand, Ma and Staunton [61] used a feature selection scheme based on correlation analysis for skin lesion classification based on a neural network, since it achieved better result than original feature-based classification. Arroyo and Zapirain [111] analysed the relevant features based on the minimum number of samples per leaf by using decision tree classifier. Several other studies have achieved good classification results by using a feature selection scheme [80, 84, 110].

Another means of determining the most discerning features based on colour and texture was addressed by Barata et al. [11], who compared the features performed by using each individual feature, all the colour features, both texture and colour features, and the best texture and colour features. The authors concluded that the colour features provide better results than the use of texture features when used individually. On the other hand, Rastgoo et al. [84] evaluated the most discerning features between shape, colour, and texture features and the evaluation revealed the potential of texture features for skin lesion classification.

\section{Skin lesion classification}

The classification step consists of recognizing and interpreting the information about the pigmented skin lesions based on features extracted from images. The classification process generally occurs by randomly dividing the available image samples in training and test sets. The training step consists of developing a classification model to be used by one or more classifiers based on the samples of the training set. Each sample is composed of features extracted from a given image and its corresponding class value, which are applied as input data to the classifier for the learning process. The testing step consists of measuring the accuracy of the model learned by the training step over the test set. In addition, such a process may present several problems concerning the dataset, such as features containing different ranges, unbalanced dataset regarding the number of samples, and/or a large number of features. Therefore, this process may require pre-processing of data, in which several methods may be applied to overcome these problems.

Feature normalization is a pre-processing step, in which methods may be applied in order to solve the problem of different ranges. The $\mathrm{z}$-score transformation is a common method used for data normalization, which allows transforming all numeric features in values within the same range, as discussed by Celebi et al. [88] and by Cavalcanti and Scharcanski [70]. Therefore, this procedure prevents the feature with range of values greater than other features from influencing the results, since several classifiers may not deal properly with different ranges.

Unbalanced datasets concerning the number of samples in each class are also a classification problem that may decrease the accuracy of the evaluation result, since the classifiers tend to be based on classes with the highest occurrence. Sampling techniques, such as over- and undersampling [140, 144], have been used to solve this problem [88, 110]. Nevertheless, random under-sampling may remove important samples, and random over-sampling may lead to over-fitting. Synthetic minority oversampling technique (SMOTE) [145] is an over-sampling techniques for overcoming the over-fitting and expanding the decision region of minority class samples. Such techniques can also be combined with ensemble methods for addressing unbalanced classes [110]. Another method to solve the unbalanced dataset problem was used by Barata et al. [11], 
in which the dataset is composed of 25 samples of melanoma and 151 samples of nevi. The authors repeated the melanoma features belonging to each training set until the same number of samples for both classes was obtained. Furthermore, they added Gaussian noise to each repeated feature set in order to prevent equal samples in the training set.

As mentioned previously, feature selection [137], which is a pre-processing step in machine learning, can be addressed to deal with datasets contain a large number of features for skin lesion classification (Sect. 3.3). The classification methods used for skin lesion diagnosis, as well as its evaluation procedures, are presented with details in following sections. Furthermore, some results of recent studies for classification of skin lesion and its patterns are also provided.

\subsection{Methods for classification}

Classification methods based on instance-based learning [140], decision trees [138], Bayesian learning [146], artificial neural networks (ANNs) [147], support vector machines (SVMs) [148], and ensemble methods [140], have been commonly applied to discriminate skin lesions in images. A description and the main advantages and disadvantages of such methods are summarized in the following, while their algorithms applied to the learning objective are presented in Table 5.

In instance-based classifiers [140], a distance function is used to assess which sample of the training set is closest to an unknown sample and then assigning the unknown sample to the class with the majority of the nearest neighbours. These classifiers have been applied due to their simplicity of implementation and their facility to deal with the existence of correlated features. In addition, new samples can be added to the training set at any time. However, they are sensitive to the existence of irrelevant features, and they require a great deal of time for classifying large datasets. Barata et al. [11] used the $k$-nearest neighbours (KNN) algorithm to classify the lesions and compared several distance functions, such as Euclidean, Kolmogorov, and Kullback-Leibler, in order to measure the distance of $\mathrm{k}$-nearest neighbours from different $k$ values. The authors concluded that it is not clear which of these three used distances is the best for such a problem, since all were considered to be the best for certain test situations. On the other hand, Rahman et al. [109] used the Bhattacharyya distance measure, since such a measure is based on the correlation between the colours and may perform better than the traditional Euclidean distance.

A decision tree [138] has a structure similar to a flowchart, in which each internal node (non-leaf) represents a test of a feature, each branch represents a result of the test,
Table 5 Classification methods applied to discriminate skin lesions from images

\begin{tabular}{|c|c|}
\hline Classification method & References \\
\hline \multicolumn{2}{|l|}{ Instance-based learning } \\
\hline KNN & {$[11,63,70,73,83,109,149]$} \\
\hline KStar & {$[108]$} \\
\hline LWL & {$[108]$} \\
\hline \multicolumn{2}{|l|}{ Decision tree } \\
\hline NBTree & {$[64,108]$} \\
\hline AD tree & {$[64]$} \\
\hline CART & {$[70,80,102,108]$} \\
\hline $\mathrm{J} 48 / \mathrm{C} 4.5 / \mathrm{C} 5.0$ & {$[10,64,77,87,106,111,150]$} \\
\hline $\mathrm{C} \& \mathrm{R}$ & [106] \\
\hline LMT & {$[10,12,24,79,151]$} \\
\hline Decision stump & {$[10,64]$} \\
\hline \multicolumn{2}{|l|}{ Bayesian network } \\
\hline BayesNet & {$[10,62,82,108]$} \\
\hline NBL & [108] \\
\hline $\mathrm{HNB}$ & {$[12]$} \\
\hline \multicolumn{2}{|l|}{ ANN } \\
\hline MLP architecture & {$[61,63,81,94,97,99,106,117]$} \\
\hline RBF network & [108] \\
\hline \multicolumn{2}{|l|}{ SVM } \\
\hline Linear kernel & {$[125]$} \\
\hline RBF kernel & {$[11,12,73,88,90,108,109,152]$} \\
\hline Polynomial kernel & {$[63,110,123]$} \\
\hline PUK kernel & {$[63]$} \\
\hline \multicolumn{2}{|l|}{ Ensemble of classifiers } \\
\hline Homogeneous ensemble & {$[110,112,153]$} \\
\hline Heterogeneous ensemble & {$[82,109]$} \\
\hline Bagging & {$[110]$} \\
\hline Random forest & {$[12,63,64,83,84]$} \\
\hline Boosting & {$[84,100,101,154]$} \\
\hline AdaBoost & {$[73,83]$} \\
\hline \multicolumn{2}{|l|}{ Other methods } \\
\hline Linear classifier & {$[93,96,105]$} \\
\hline Regression analysis & {$[104,108]$} \\
\hline Prototype based & {$[82]$} \\
\hline Discriminant analysis & {$[80,102,155]$} \\
\hline Maximum likelihood & {$[62,109,149]$} \\
\hline
\end{tabular}

$K N N k$-nearest neighbour, $L W L$ locally weighted learning, NBTree naïve Bayes/decision tree, $A D$ tree alternative decision tree, $C A R T$ classification and regression trees, $C \& R$ classification and regression, $L M T$ logistic model tree, $R F$ random forest, $N B L$ naïve Bayes multinomial, $H N B$ hidden naïve Bayes, $A N N$ artificial neural network, $M L P$ multilayer perceptron, $R B F$ radial basis function, $S V M$ support vector machine, PUK Pearson VII function-based universal kernel

and each external node (leaf) indicates a prediction of the class. Several methods based on decision trees have been frequently applied to classify skin lesions [10, 12, 24, 77, 106, 108]. Understanding such a structure, 
as well as ease of rule generation, is quite straightforward. However, the excess of adjustments (over-fitting) and the difficulties in dealing with correlated features are the major drawbacks of decision trees.

Bayesian learning-based methods [146] compute the probability of a given set of features to belong to each class, assuming that the features are independent. These methods have been applied to classify skin lesions particularly because of their fast training [10, 12, 108]. Although Bayesian methods provide fast training and no sensitivity to irrelevant features, they assume that the features need to be independent, which can be a disadvantage of these methods.

ANNs [147] are parallel distributed systems composed of layers of input and output elements linked by weighted connections. During the learning phase, the weights are adjusted to predict the correct class based on the input samples. The multilayer perceptron (MLP) is one of the most applied architectures of ANNs [81, 106], since such architecture presents good capability and flexibility to solve several non-separable problems. This architecture may include one or more layers of processing, also called hidden layers, placed between the input and output layers. The back-propagation is a supervised learning algorithm widely used in the MLP architecture [61], which consists of forward and backward processes applied to adjust the weight values of the connections. Although ANNs have been proposed to solve many pattern recognition problems, these classifiers may have long training time depending on the size of the training set.

SVMs [148] involve a method based on statistical learning applied to building a hyper-plane to separate the data according to the defined classes. This kind of classifier has been commonly applied to classify skin lesions due to its good generalization properties. Furthermore, kernel functions simplify the process of separating the nonlinear data by using a simple hyper-plane in a high dimension feature space. However, these classifiers are sensitive to noise, and the classification process is based on a binary class. The radial basis function (RBF) kernel has been commonly adopted in several studies $[11,12,108]$ due to the several advantages compared to other kernels, such as: greater stability compared to the polynomial kernel and reduced number of hyper-parameters that need to be established compared to the polynomial and sigmoid kernels [88].

The ensemble methods [140] have been recently adopted to diagnose skin lesions [82-84, 110, 156]. Ensemble models may be constructed with either several classification algorithms, classified as heterogeneous, or only with one classification algorithm, classified as homogeneous, which can be developed through data manipulation [157]. Average, weighted average, sum, product, maximum, minimum, and median are some examples of integration strategies based on the outputs of classifiers. Voting methods from the candidates of a rank may also be used for this same purpose. The common algorithms applied to manipulate the training samples are the bagging and boosting algorithms [157]. Random forest [158] and AdaBoost [159] are also popular ensemble methods. Random forest is a variation of the Bagging algorithm that is used to create individual decision trees, whereas AdaBoost is a popular boosting algorithm that maintains a set of weighting systems over the training samples. Ensemble methods consist of combining the results of several classification models in order to develop a more robust system that provides more accurate results than by using a single classifier. However, such methods can present a high computational complexity.

\subsection{Evaluating the classification}

The main objective of the classification process of skin lesions is to achieve good results for distinguishing between different lesion classes. In order to fulfil this purpose, several classification models based on different feature subsets, samples, and classifiers are evaluated by using test sets. Therefore, new samples are classified, and the predicted class is compared to the known class to evaluate the classification performance. Among several evaluation procedures, the cross-validation (XVAL) procedure [140] is the most commonly used in the literature to evaluate the results of skin lesion classification, since it avoids over-fitting while testing the capacity of the classifier to generalize. The $k$-fold cross-validation $[12,108]$ and leave-one-out $[11,93]$ are examples of cross-validation procedures proposed for classifying skin lesions in images. The half-and-half test is another evaluation procedure, which was applied by Iyatomi et al. [96]. In addition, the authors evaluated the performance of classifiers using tenfold cross-validation, leave-one-out cross-validation and half-and-half tests, and they concluded that the results are almost equivalent and may be considered reasonable.

Statistical measures based on performance metrics [160] are computed to compare the performance of one or several classification models according to the outcomes of classifiers. Some possible outcomes of classifiers based on the predicted class and known class are: (1) true positive (TP), (2) true negative (TN), (3) false positive (FP), and (4) false negative (FN). These outcomes represent the number of correct (true) and incorrect (false) classification for each class (positive and negative). For example, in a classification process between two classes, one class may be considered positive and another negative. Usually, the positive samples represent the most important class to classify (e.g. skin cancer), and benign lesion stands for the negative 
samples. Therefore, the TP rate is the number of correctly classified positive samples, the TN rate is the number of correctly classified negative samples, the FP rate is the number of incorrectly classified negative samples, and the FN rate is the number of incorrectly classified positive samples.

The aforementioned rates may be represented by a confusion matrix, which is the basis for several metrics used by researchers to measure the performance of the classification [10, 12, 81], such as: (1) the precision that is the percentage of correctly classified samples for each given class with respect to its true and false predictions, (2) the recall or sensitivity, which is the percentage of correctly classified positive samples with respect to all positive samples, (3) the specificity, which is the percentage of correctly classified negative samples with respect to all negative samples, and (4) the accuracy that is the percentage of correctly classified positive and negative samples based on all samples. Area under the ROC curve (AUC) is an additional term associated with the receiver operating characteristics (ROC) graph [160], which is also used to compare the performance of the classification, since it is a very useful tool for visualizing and evaluating classifiers $[10,11,81]$. Currently, such measure is commonly used and is able to provide a more robust classification performance measure than other evaluation measures [160].

\subsection{Skin lesion classification performance}

For the skin lesion classification process, one or several techniques have been evaluated to achieve the best results. The performance of such a process depends on several issues, such as the segmented image, and extracted or selected features, as well as the classification method used. The classification process may be binary or multiclass and includes different classes according to the classification goal, such as: (1) malignancy of the lesions (benign vs. malignant) $[12,106]$ and (2) distinct types of skin lesions (melanoma vs. nevus [94, 108], melanocytic vs. non-melanocytic [93], and dysplastic vs. non-dysplastic vs. melanotic [108]). Furthermore, skin lesion features are also classified in terms of: (1) border features (regular vs. irregular [90, 106] and irregularity level [95]), (2) presence of main colours existing in malignant lesions [81, 112], (3) presence of features of the seven-point checklist $[24,79,161]$, (4) presence of global patterns [72, 73, 162], and (5) presence of local patterns [71, 128].

Table 6 summarizes the best results of recent studies concerning skin lesion classification. The table indicates the number and type of image used, the techniques employed in the segmentation step and feature selection, the number of extracted and selected features, the classification algorithms and the values of the evaluation measures used. The performance of several classifiers has been compared, e.g. in terms of the discrimination between benign lesions and melanomas, by several authors.

Zortea et al. [80] compared the classification performance of quadratic discriminant analysis (QDA), LDA, and CART and obtained the best results with QDA. In the study of Rastgoo et al. [84], better results were achieved using a random forest than a gradient boosting and SVM classifier. Likewise, Barata et al. [83] have also obtained the best results by applying a random forest than using AdaBoost, SVM, and KNN. Schaefer et al. [110] proposed an ensemble method based on a SVM (polynomial kernel), non-pairwise measure of diversity (fuzzy Shannon), and neural network based on classifier fusion, which obtained the best results when compared to other ensemble methods, as well as with individual SVM classifier.

Ensemble methods have performed better than individual classifiers in several studies [11, 109], whereas Alcón et al. [10] obtained the best results in both the individual logistic model tree (LMT) classifier and AdaBoost ensemble method. Meanwhile, the authors considered the LMT classifier more useful due to the complexity computation of the ensemble model. Consequently, there is no ideal method to solve all problems in skin lesion classification, as may be observed in findings in the literature. The performance of the classification relies on several conditions, mainly on discriminative features, as previously discussed.

The features extracted from the lesion have also been used for pattern detection or classification in order to assist in skin lesion diagnosis. Table 7 summarizes the best results of recent studies concerning global and local pattern classification in dermoscopic images. The table indicates the number of image used, the target of the detection or classification, and the values of the evaluation measures.

Several methods have been proposed for the pattern analysis task in skin lesion diagnosis. Some of these methods have also used feature selection techniques, and the performance of several classifiers has also been taken into account $[62,63,73]$. One concern in this task is in identifying the presence of global patterns, since few studies have been done on such patterns in automatic diagnosis of skin lesions. To the best of our knowledge, only one study dealing with the classification of all global patterns of skin lesions has been proposed [73], and no previous study has addressed the issue to identify the absence of such patterns. Indeed, it should be noted that the multicomponent pattern and the absence of patterns can indicate a higher probability of being a malignant lesion.

Abbas et al. [73] proposed the classification of skin lesion global patterns by using AdaBoost algorithm based on colour and texture properties from a perceptually 


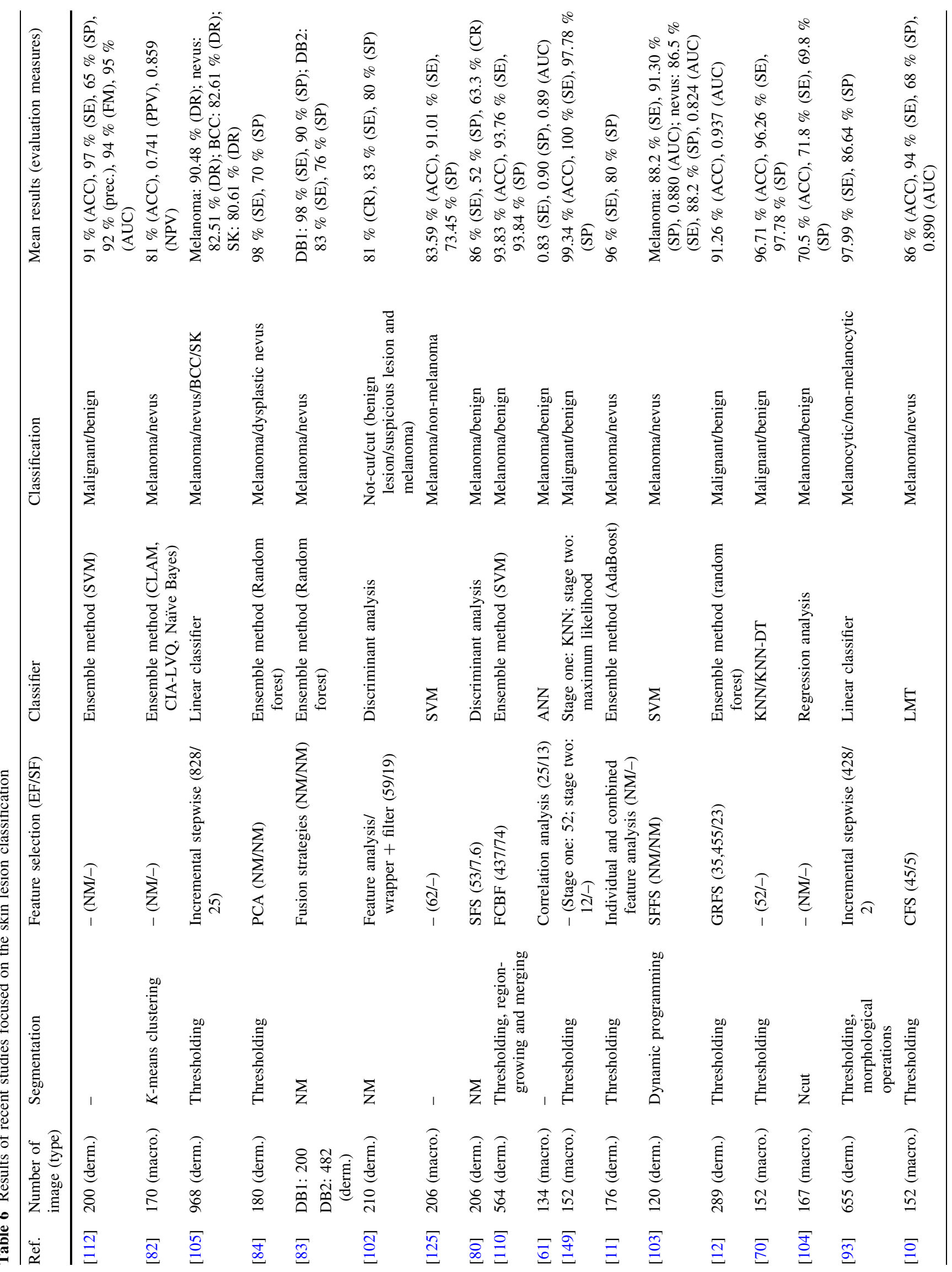




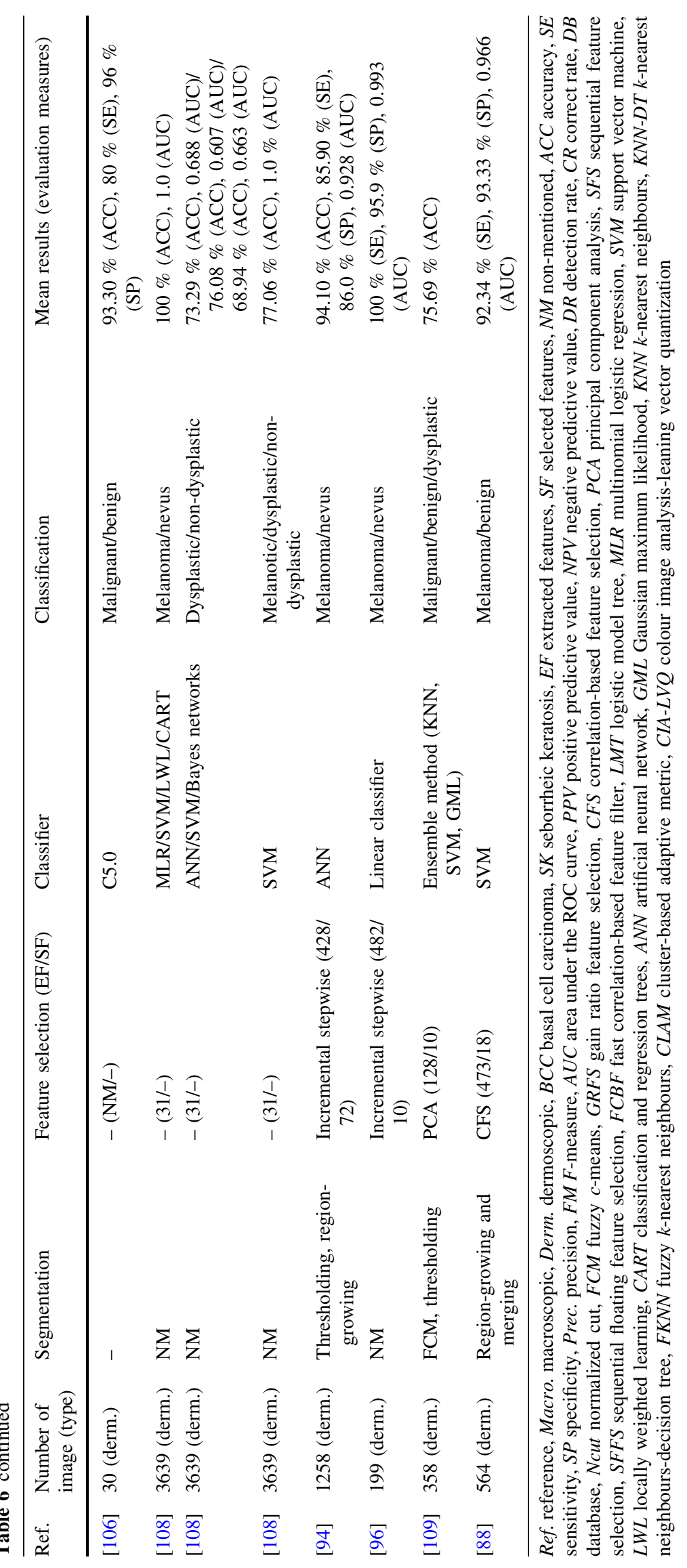


Table 7 Results of recent studies focused on the global and local pattern analysis in dermoscopic images

\begin{tabular}{|c|c|c|c|}
\hline References & $\begin{array}{l}\text { Number } \\
\text { of images }\end{array}$ & Detection/classification & Mean results (evaluation measures) \\
\hline \multicolumn{4}{|c|}{ Global pattern } \\
\hline [73] & 350 & Reti./Glob./Cob./Homo./Paral./Starb./Mult. & $89.28 \%$ (SE), $93.75 \%$ (SP), 0.986 (AUC) \\
\hline$[162]$ & 180 & Reti./Glob./Cob./Homo./Paral./Starb./ & $93.08 \%$ (SE), $91.45 \%$ (SP), 0.948 (AUC) \\
\hline [72] & 325 & Reti./Glob./Cob./Homo./Paral./ & $86.8 \%(\mathrm{ACC})$ \\
\hline [74] & 160 & Reti.; Glob./ & $89 \%$ (ACC); $95 \%$ (ACC) \\
\hline [71] & 360 & Mult. & NM \\
\hline [98] & 100 & Reti./Glob./Cob./Homo./Paral./ & $94 \%(\mathrm{ACC})$ \\
\hline [124] & 100 & Reti./Glob./Cob./Homo./Paral./ & $86 \%(\mathrm{ACC})$ \\
\hline [96] & 213 & Paral. ridge; paral. furrow; fibrillar & 0.985 (AUC); 0.931 (AUC); 0.890 (AUC) \\
\hline$[121]$ & 44 & Reti./Glob./Homo./ & $94(\mathrm{ACC})$ \\
\hline \multicolumn{4}{|c|}{ Pigmented network } \\
\hline [112] & NM & Typical & $74 \%$ (ACC), 0.82 (AUC), $79 \%$ (Prec.) \\
\hline$[163]$ & 122 & Present/absent; typical/atypical & $85 \%$ (ACC), 0.821 (AUC); $100 \%$ (ACC) \\
\hline [111] & 220 & Present/absent & $86 \%$ (SE), $81.67 \%$ (SP) \\
\hline$[100]$ & 200 & Present/absent & $86.2 \%$ (ACC), $91.1 \%$ (SE), $82.1 \%$ (SP) \\
\hline$[62]$ & 734 & Present/absent & $0.922(\mathrm{AUC})$ \\
\hline [71] & 360 & Melanoma/benign & NM \\
\hline$[24]$ & 115 & Atypical/absent & $80 \%$ (SE), $82 \%$ (SP) \\
\hline [128] & NM & Present/absent & NM \\
\hline$[101]$ & 436 & Present/absent; absent/typical/atypical & $\begin{array}{l}93 \% \text { (ACC), } 0.935 \text { (Prec.), } 0.933 \text { (Rec.); } \\
82 \% \text { (ACC), } 0.820 \text { (Prec.), } 0.823 \text { (Rec.) }\end{array}$ \\
\hline$[64]$ & 106 & Melanoma/benign & $95.4 \%(\mathrm{ACC})$ \\
\hline$[164]$ & 173 & Typical/atypical & $85 \%(\mathrm{ACC})$ \\
\hline [165] & 60 & No network/partial/complete & $88.3 \%(\mathrm{ACC})$ \\
\hline [166] & 30 & Typical/atypical & NM \\
\hline$[122]$ & 155 & Present/absent & $80 \%(\mathrm{ACC})$ \\
\hline [155] & NM & Present/absent & NM \\
\hline \multicolumn{4}{|c|}{ Dots/globules } \\
\hline [112] & NM & Absent; typical; atypical & $\begin{array}{l}47 \% \text { (ACC), } 0.53 \text { (AUC), } 47 \% \text { (Prec.); } \\
70 \% \text { (ACC), } 0.55 \text { (AUC), } 39 \% \text { (Prec.); } \\
61 \% \text { (ACC), } 0.51 \text { (AUC), } 29 \% \text { (Prec.) }\end{array}$ \\
\hline$[63]$ & 108 & Malignant/non-malignant & 0.903 (ACC), 0.884 (SE), 0.923 (SP) \\
\hline [128] & NM & Present/absent & NM \\
\hline [71] & 360 & Melanoma/benign & NM \\
\hline [155] & NM & Present/absent & NM \\
\hline \multicolumn{4}{|l|}{ Streaks } \\
\hline [112] & NM & Absent & $85 \%$ (ACC), 0.79 (AUC), $95 \%$ (Prec.) \\
\hline [154] & 945 & Present/absent; regular/irregular; absent/regular/irregular & $\begin{array}{c}78.3 \% \text { (ACC), } 83.2 \% \text { (AUC); } 83.6 \% \text { (ACC), } \\
88.9 \% \text { (AUC); } 76.1 \% \text { (ACC), } 85 \% \text { (AUC) }\end{array}$ \\
\hline [152] & 99 & Absent/regular/irregular & $91 \%(\mathrm{ACC})$ \\
\hline$[151]$ & 53 & Present/absent & $86 \%$ (SE), $88 \%$ (SP) \\
\hline$[24]$ & 200 & Irregular/absent & $86 \%$ (SE), $88 \%$ (SP) \\
\hline [78] & 10 & Present/absent & NM \\
\hline \multicolumn{4}{|c|}{ Blue-whitish veil } \\
\hline [112] & NM & Absent & $90 \%$ (ACC), 0.96 (AUC), $99 \%$ (Prec.) \\
\hline [118] & $200 ; 100$ & Present/absent & $87 \%$ (ACC); $67 \%$ (ACC) \\
\hline$[150]$ & 887 & Present/absent & $80.50 \%$ (SE), $90.93 \%$ (SP) \\
\hline [74] & 160 & Present/absent & $86 \%(\mathrm{ACC})$ \\
\hline [71] & 360 & Melanoma/benign & NM \\
\hline
\end{tabular}


Table 7 continued

\begin{tabular}{|c|c|c|c|}
\hline References & $\begin{array}{l}\text { Number } \\
\text { of images }\end{array}$ & Detection/classification & Mean results (evaluation measures) \\
\hline [24] & 110 & Present/absent & $90 \%(\mathrm{SE}), 93 \%(\mathrm{SP})$ \\
\hline [151] & 135 & Present/absent & $87 \%$ (SE), $85 \%$ (SP) \\
\hline [77] & $100 ; 545$ & Present/absent; melanoma/benign & $\begin{array}{l}84.33 \% \text { (SE), } 96.19 \% \text { (SP); } \\
69.35 \% \text { (SE), } 89.97 \% \text { (SP) }\end{array}$ \\
\hline \multicolumn{4}{|l|}{ Blotches } \\
\hline$[24]$ & 110 & Irregular/absent & $87 \%$ (SE), $90 \%$ (SP) \\
\hline [99] & 424 & Melanoma/benign & $81.2 \%(\mathrm{ACC})$ \\
\hline [120] & 50 & Present/absent & NM \\
\hline [117] & 512 & Melanoma/benign & $77 \%$ (ACC) \\
\hline \multicolumn{4}{|c|}{ Hypopigmentation } \\
\hline [97] & 244 & Melanoma/nevus & $0.952(\mathrm{AUC})$ \\
\hline \multicolumn{4}{|c|}{ Regression structures } \\
\hline [112] & NM & Absent & $89 \%$ (ACC), 0.86 (AUC), $98 \%$ (Prec.) \\
\hline [24] & 110 & Present/absent & $80 \%$ (SE), $83 \%$ (SP) \\
\hline$[151]$ & 80 & Present/absent & $80 \%$ (SE), $83 \%$ (SP) \\
\hline \multicolumn{4}{|c|}{ Vascular structures } \\
\hline [166] & NM & Present/absent & NM \\
\hline
\end{tabular}

The references of research about local features also include the works focused on the seven-point checklist method

$N M$ non-mentioned, ACC accuracy, SE sensitivity, SP specificity, AUC area under the ROC curve, Prec. precision, Rec. recall, Reti. reticular, Glob. globular, Cob. cobblestone, Homo. homogeneous, Paral. parallel, Starb. starburst, Multi. multicomponent

uniform colour space. Furthermore, the authors developed a multilabel learning algorithm (AdaBoost.MC) to solve the problem of multicomponent pattern. This pattern is determined by fusing the results produced by AdaBoost.MC based on maximum a posteriori (MAP) and robust ranking principles. The method achieved superior results compared with the multilabel SVM and KNN.

Local pattern detection of dermoscopy images is a challenging task to assist in discriminating between benign and malignant skin lesions. The presence of local patterns, such as blue-whitish veil and regression structures, or even some patterns considered atypical, irregular, or asymmetric, may identify a malignant lesion. To the best of our knowledge, no previous study has dealt with all skin lesion local patterns. Leo et al. [24] proposed a method based on LMT to classify five local patterns based on the sevenpoint checklist method. The authors segmented the lesion colour by using PCA, 2D histogram construction, peakpicking algorithm, and histogram and lesion partitioning, in order to detect a blue-whitish veil, irregular pigmentation, and regression structures. In addition, the authors combined structural and spectral methods to extract texture features, such as median filter, close-opening operation, fast Fourier transform (FFT), high-pass filtering, inverse fast Fourier transform (IFFT) and suitable thresholding, in order to detect the atypical pigment network, and irregular streaks. The authors achieved good results in the detection of such local patterns.
Most studies have been proposed for the pigmented network detection [100, 111, 163]. In addition, other studies have considered feature extracted from patterns for discriminating between benign and malignant skin lesions [63, 64, 77]. Maglogiannis and Delibasis [63] classified the skin lesion into malignant and non-malignant and achieved superior results with inclusion of the dot-related features to the lesion-related features. The SVM classifier (polynomial kernel) yielded better results than MLP, KNN, random forest, and SVM (PUC kernel) based on dot-related features. The dots were segmented using a circularity function and definition of diffusivity after enhancing dark circular structures using inverse nonlinear diffusion.

\section{Discussion}

Dermoscopic images have been widely used for diagnosis of pigmented skin lesions [167, 168], since they allow suitable visualization with more details of pigmentation patterns on the surface of the lesion. Furthermore, previous clinical studies have addressed an increase in sensitivity of the melanoma diagnosis by dermoscopic compared to diagnosis by macroscopic image [169]. Among the several skin lesion diagnostic methods using dermoscopic images [67], the ABCD rule has been commonly applied to extract features for computational analysis [84, 149]. This rule allows for easy understanding and provides simplicity of 


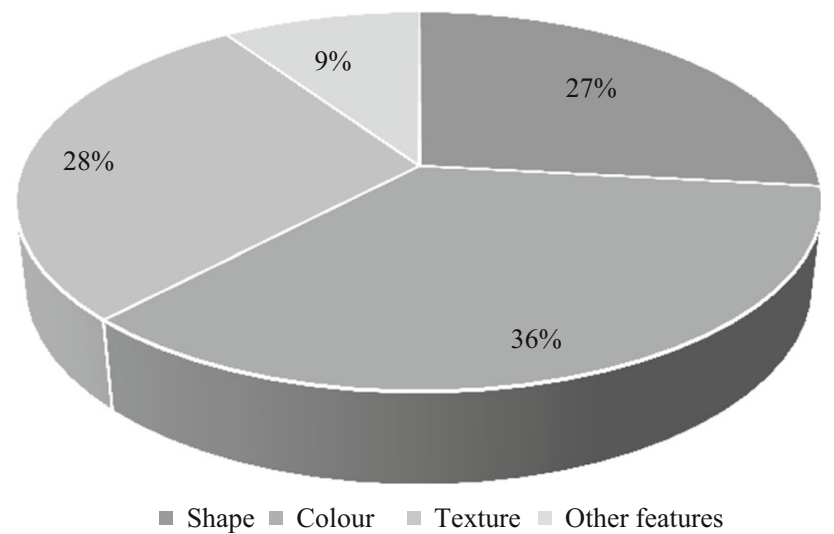

Fig. 4 Distribution of the reviewed skin lesion classification methods according to the main feature used

application while showing reliable results for the melanoma diagnosis. On other hand, previous clinical studies [69] reported that methods based on pattern analysis performed better than the ABCD rule for the diagnosis of melanocytic skin lesions. In recent years, descriptors mainly based on shape, colour, and texture have been proposed to identify and classify patterns in skin lesion images, as well as to discriminate benign and malignant lesions. Pattern analysis of pigmented skin lesions has shown promising results and may continue to be the focus of intense research in the coming years [73, 81]. Figure 4 illustrates the distribution of the methods that have been proposed for skin lesion classification reviewed in this article according to the main feature used.

The classification process of skin lesions in images must be effective, since it is crucial to assist dermatologists in the diagnosis of these lesions by means of CAD systems. In addition, the evaluation and improvement of the performance of classifiers are essential for the pattern recognition research field [58]. A relevant problem that affects the performance of classifiers is the definition of the meaningful features for representing the classes. Consequently, the feature extraction and selection steps are very important to achieve better performance for the computational diagnosis of skin lesions in images. The application of several descriptions may be required considering the large number of features extracted from images. For dealing with this issue, feature selection methods have been applied to establish the most relevant features [61, 80, 84, 110], since these methods allow removing the redundant and/or irrelevant features. As a consequence, the feature extraction time, the training and testing computational load, and the classification complexity are all reduced, while the classification performance may be improved. The result of the feature selection process depends on the search strategy and evaluation model applied as well as their established parameters. In regard to the classification process, the performance depends on several factors, such as

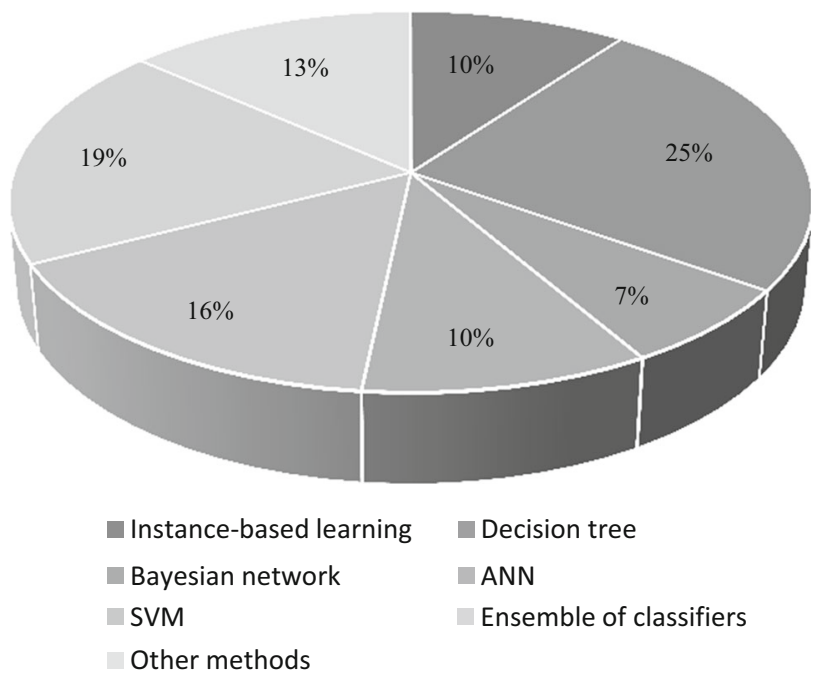

Fig. 5 Distribution of the classification algorithms used by the reviewed methods for skin lesion classification

the extracted and selected features, established parameters and chosen classification method. The classification algorithms should be chosen based on the classification problem and available data regarding advantages and disadvantages of each algorithm. Figure 5 illustrates the distribution of the classification algorithms used in the methods reviewed in this article for skin lesion classification.

Classification methods based on a decision tree have been used by many authors for the skin lesion classification $[10,12,24]$. The simplicity of the structure in terms of ease of understanding and visualization, as well as the easy rule generation, is one of the important advantages of this method. Ensemble methods [140], which aim to combine the strengths of different classifiers, have also been commonly proposed to improve the performance of the classification of skin lesions. These methods have performed better than individual classifiers [11, 110]. The SVM classifier [148] has also been applied to discriminate skin lesions due to its good generalization and simplification of the nonlinear data separation by means of kernel functions $[63,73]$. Despite the long training time, ANNs have been proposed in various studies $[61,81,106]$ to deal with complex pattern recognition problems. Recently, the linear classifier [93], regression analysis [104], prototype-based classifier [82], discriminant analysis [80], and maximum likelihood [149], have also been proposed to solve problems of skin lesion classification.

\section{Conclusion and future trends}

Pigmented skin lesion classification is an area of great research interest due to its importance in skin cancer prevention, as well as in the early diagnosis. This review 
provides an overview of current developments of computational methods for skin lesion image classification. Studies specifically addressing automatic methods applied to the feature selection and extraction steps, based on several clinical approaches, were presented in this review. In addition, the skin lesion classification step was addressed by including classifiers and evaluation procedures, as well as some performance results for pattern and lesion classification.

From this review, one may conclude that several studies focused on skin lesion classification have been proposed for use in CAD systems. Such systems aim at an effective computational diagnosis of pigmented skin lesions to assist dermatologists in their diagnosis. Although this research topic has been addressed in several studies, resulting in successful systems, new methodologies may be proposed to fill gaps that still have not been fully addressed, as well as to improve the performance of existing methods. Most studies involve extraction of several features from dermoscopic images and comparison of two or more classification methods to identify benign and malignant lesions. However, some studies used feature selection methods to achieve a better classification performance. Detection and classification of skin lesion patterns have also been the goal in several studies. Recently, global and local pattern recognition has been of great interest to researchers.

In conclusion, future trends regarding image computational analysis of pigmented skin lesions involve searching for new methods aiming to develop more efficient and effective expert systems for the computational diagnosis based on macroscopic and dermoscopic images. Hence, several issues may be addressed to achieve this goal, in particular: (1) the evolution features may be better explored in order to develop methods to analyse changes in size, shape, shades of colour, and surface features on skin lesions-extracted features based on evolution criterion along with the other criteria features may complement the diagnosis; (2) the development and evaluation of new computational methods to identify the presence of global patterns, mainly the starburst and multicomponent patterns, since few studies have explored such patterns; (3) the lack of computational methods to detect some skin lesion local patterns and access their irregularity that can also be important to assist in diagnosis of specific lesions; (4) the development of new approaches for colour and asymmetry patterns, and positive feature analysis based on Menzies's method is important for future applications of this method for computational diagnosis of skin lesions; (5) in order to find more relevant features for the given problem, different feature selection models may be compared; and (6) the evaluation of new classifiers, ensemble models and parameter optimisation need to be addressed in order to classify skin lesions and to improve on the current results.
Computational methods based on the issues aforementioned may perform better and more effectively in diagnosing skin lesions in images. In addition, such methods may cover several problems regarding skin lesion classification, which convert $\mathrm{CAD}$ systems into more complete expert systems for diagnosing such lesions based on macroscopic and dermoscopic images.

Acknowledgments The first author would like to thank the $\mathrm{CNPq}$ ("Conselho Nacional de Desenvolvimento Científico e Tecnológico"), in Brazil, for her PhD grant. This work is funded by European Regional Development Funds (ERDF), through the Operational Programme "Thematic Factors of Competitiveness" (COMPETE), and Portuguese Funds, through "Fundação para a Ciência e a Tecnologia (FCT)", under the Project: FCOMP-01-0124-FEDER028160/PTDC/BBB-BMD/3088/2012. Authors gratefully acknowledge the funding of Project NORTE-01-0145-FEDER-000022-SciTech-Science and Technology for Competitive and Sustainable Industries, cofinanced by "Programa Operacional Regional do Norte" (NORTE2020), through "Fundo Europeu de Desenvolvimento Regional" (FEDER).

\section{References}

1. Razmjooy N, Mousavi BS, Soleymani F, Khotbesara MH (2013) A computer-aided diagnosis system for malignant melanomas. Neural Comput Appl 23(7):2059-2071

2. Ruela M, Barata C, Marques JS, Rozeira J (2015) A system for the detection of melanomas in dermoscopy images using shape and symmetry features. Comput Methods Biomech Biomed Eng Imaging Vis. doi:10.1080/21681163.2015.1029080

3. Scharcanski J, Celebi ME (2013) Computer vision techniques for the diagnosis of skin cancer. Springer, Berlin

4. INCA (2014) Estimativa 2014: Incidência de Câncer no Brasil. Instituto Nacional de Câncer José Alencar Gomes da Silva, Coordenação de Prevenção e Vigilância. INCA, Rio de Janeiro

5. American Cancer Society (2014) Cancer facts \& figures 2014. American Cancer Society, Atlanta

6. Cancer Research UK (2013) Cancer statistic report on skin cancer. Cancer Research, London, UK. http://www.cancerresearchuk.org/ cancer-info/cancerstats/types/skin/?script=true. Accessed 01 Mar 2016

7. Bourne P, Cameron A, Gourhant J-Y, Hackett T, Hlaing W, Kittler H, McColl I, Minas S, Rosendahl C (2007) The international atlas of dermoscopy and dermatoscopy. The Skin Cancer Society of Australia, Australia. http://www.dermosco pyatlas.com/index.cfm. Accessed 01 Mar 2016

8. Smith L, MacNeil S (2011) State of the art in non-invasive imaging of cutaneous melanoma. Skin Res Technol 17(3):257-269

9. Cavalcanti PG, Scharcanski J (2013) Macroscopic pigmented skin lesion segmentation and its influence on lesion classification and diagnosis. In: Celebi ME, Schaefer G (eds) Color medical image analysis. Springer, Dordrecht, pp 15-39

10. Alcón JF, Ciuhu C, Ten Kate W, Heinrich A, Uzunbajakava N, Krekels G, Siem D, de Haan G (2009) Automatic imaging system with decision support for inspection of pigmented skin lesions and melanoma diagnosis. IEEE J Sel Top Signal Process 3(1): 14-25

11. Barata C, Ruela M, Francisco M, Mendonça T, Marques JS (2013) Two systems for the detection of melanomas in dermoscopy images using texture and color features. IEEE Syst $\mathbf{J}$ 8(3):965-979 
12. Garnavi R, Aldeen M, Bailey J (2012) Computer-aided diagnosis of melanoma using border- and wavelet-based texture analysis. IEEE Trans Inf Technol Biomed 16(6):1239-1252

13. Oliveira RB, Filho ME, Ma Z, Papa JP, Pereira AS, Tavares JMRS (2016) Computational methods for the image segmentation of pigmented skin lesions: a review. Comput Methods Programs Biomed 131:127-141

14. Silveira M, Nascimento JC, Marques JS, Marcal ARS, Mendonca T, Yamauchi S, Maeda J, Rozeira J (2009) Comparison of segmentation methods for melanoma diagnosis in dermoscopy images. IEEE J Sel Top Signal Process 3(1):35-45

15. Wong A, Scharcanski J, Fieguth P (2011) Automatic skin lesion segmentation via iterative stochastic region merging. IEEE Trans Inf Technol Biomed 15(6):929-936

16. Yuksel ME, Borlu M (2009) Accurate segmentation of dermoscopic images by image thresholding based on type-2 fuzzy logic. IEEE Trans Fuzzy Syst 17(4):976-982

17. Zhou H, Schaefer G, Celebi ME, Iyatomi H, Norton K, Liu T, Lin F (2010) Skin lesion segmentation using an improved snake model. In: Annual international conference of the engineering in Medicine and Biology Society, Buenos Aires, August 31September 4. IEEE, pp 1974-1977

18. Zhou H, Li X, Schaefer G, Celebi ME, Miller P (2013) Mean shift based gradient vector flow for image segmentation. Comput Vis Image Underst 117(9):1004-1016

19. Zhou H, Schaefer G, Celebi ME, Lin F, Liu T (2011) Gradient vector flow with mean shift for skin lesion segmentation. Comput Med Imaging Graph 35(2):121-127

20. Abbas Q, Celebi ME, Garcia IF (2012) A novel perceptuallyoriented approach for skin tumor segmentation. Int J Innov Comput Inf Control 8(3):1837-1848

21. Abbas Q, Fondón I, Rashid M (2011) Unsupervised skin lesions border detection via two-dimensional image analysis. Comput Methods Programs Biomed 104(3):e1-e15

22. Norton K, Iyatomi H, Celebi ME, Schaefer G, Tanaka M, Ogawa K (2010) Development of a novel border detection method for melanocytic and non-melanocytic dermoscopy images. In: Annual international conference of the IEEE Engineering in Medicine and Biology Society, Buenos Aires, August 31-September 4. IEEE, pp 5403-5406

23. Norton K-A, Iyatomi H, Celebi ME, Ishizaki S, Sawada M, Suzaki R, Kobayashi K, Tanaka M, Ogawa K (2012) Threephase general border detection method for dermoscopy images using non-uniform illumination correction. Skin Res Technol 18(3):290-300

24. Leo GD, Paolillo A, Sommella P, Fabbrocini G (2010) Automatic diagnosis of melanoma: a software system based on the 7 -point check-list. In: International conference on system sciences, Hawaii, January 5-8, pp 1-10

25. Garnavi R, Aldeen M, Celebi ME, Varigos G, Finch S (2011) Border detection in dermoscopy images using hybrid thresholding on optimized color channels. Comput Med Imaging Graph 35(2):105-115

26. Abbas Q, Garcia IF, Celebi ME, Ahmad W, Mushtaq Q (2013) A perceptually oriented method for contrast enhancement and segmentation of dermoscopy images. Skin Res Technol 19(1):e490-e497

27. Flores E, Scharcanski J (2016) Segmentation of melanocytic skin lesions using feature learning and dictionaries. Expert Syst Appl 56:300-309

28. Ma Z, Tavares JMRS (2016) A novel approach to segment skin lesions in dermoscopic images based on a deformable model. IEEE J Biomed Health Inform 20(2):615-623

29. Celebi ME, Wen Q, Hwang S, Iyatomi H, Schaefer G (2013) Lesion border detection in dermoscopy images using ensembles of thresholding methods. Skin Res Technol 19(1):e252-e258
30. Abbas Q, Celebi ME, García IF (2012) Skin tumor area extraction using an improved dynamic programming approach. Skin Res Technol 18(2):133-142

31. Abbas Q, Celebi ME, Fondón García I, Rashid M (2011) Lesion border detection in dermoscopy images using dynamic programming. Skin Res Technol 17(1):91-100

32. Garnavi R, Aldeen M, Celebi ME (2011) Weighted performance index for objective evaluation of border detection methods in dermoscopy images. Skin Res Technol 17(1):35-44

33. Celebi ME, Schaefer G, Iyatomi H, Stoecker WV, Malters JM, Grichnik JM (2009) An improved objective evaluation measure for border detection in dermoscopy images. Skin Res Technol 15(4):444-450

34. Zhou H, Schaefer G, Sadka AH, Celebi ME (2009) Anisotropic mean shift based fuzzy c-means segmentation of dermoscopy images. IEEE J Sel Top Signal Process 3(1):26-34

35. Celebi ME, Aslandogan YA, Stoecker WV, Iyatomi H, Oka H, Chen X (2007) Unsupervised border detection in dermoscopy images. Skin Res Technol 13(4):454-462

36. Cavalcanti PG, Scharcanski J, Lopes CBO (2010) Shading attenuation in human skin color images. In: 6th international symposium on visual computing, Las Vegas, November 29December 1. Springer, Berlin, pp 190-198

37. Glaister J, Amelard R, Wong A, Clausi D (2013) MSIM: multistage illumination modeling of dermatological photographs for illumination-corrected skin lesion analysis. IEEE Trans Biomed Eng 60(7):1873-1883

38. Schaefer G, Rajab MI, Celebi ME, Iyatomi H (2011) Colour and contrast enhancement for improved skin lesion segmentation. Comput Med Imaging Graph 35(2):99-104

39. Celebi ME, Iyatomi H, Schaefer G (2009) Contrast enhancement in dermoscopy images by maximizing a histogram bimodality measure. In: 16th IEEE international conference on image processing, Cairo, November 7-10. IEEE, pp 2601-2604

40. Beuren AT, Janasieivicz R, Pinheiro G, Grando N, Facon J (2012) Skin melanoma segmentation by morphological approach. In: International conference on advances in computing, communications and informatics, Chennai, August 3-5. ACM, pp 972-978

41. Barata C, Celebi ME, Marques JS (2015) Improving dermoscopy image classification using color constancy. IEEE J Biomed Health Inform 19(3):1146-1152

42. Barata C, Celebi ME, Marques JS (2015) Towards a robust analysis of dermoscopy images acquired under different conditions. In: Celebi ME, Mendonca T, Marques JS (eds) Dermoscopy image analysis. CRC Press, Boca Raton, pp 1-22

43. Abbas Q, Garcia IF, Celebi ME, Ahmad W, Mushtaq Q (2013) Unified approach for lesion border detection based on mixture modeling and local entropy thresholding. Skin Res Technol 19(3):314-319

44. Barcelos CAZ, Pires VB (2009) An automatic based nonlinear diffusion equations scheme for skin lesion segmentation. Appl Math Comput 215(1):251-261

45. Celebi ME, Iyatomi H, Schaefer G, Stoecker WV (2009) Approximate lesion localization in dermoscopy images. Skin Res Technol 15(3):314-322

46. Zhou H, Chen M, Gass R, Rehg JM, Ferris L, Ho J, Drogowski L (2008) Feature-preserving artifact removal from dermoscopy images. In: Proceedings of the SPIE medical imaging 2008 conference, San Diego, February 16-21. International Society for Optics and Photonics, pp 69141B-1-69141B-9

47. Wighton P, Lee TK, Atkins MS (2008) Dermascopic hair disocclusion using inpainting. In: Proceedings of the SPIE medical imaging 2008 conference, San Diego, February 16-21. International Society for Optics and Photonics, pp 6914271-691427-8 
48. Xie F-Y, Qin S-Y, Jiang Z-G, Meng R-S (2009) PDE-based unsupervised repair of hair-occluded information in dermoscopy images of melanoma. Comput Med Imaging Graph 33(4):275-282

49. Kiani K, Sharafat AR (2011) E-shaver: an improved DullRazor ${ }^{\circledR}$ for digitally removing dark and light-colored hairs in dermoscopic images. Comput Biol Med 41(3):139-145

50. Abbas Q, Celebi ME, García IF (2011) Hair removal methods: a comparative study for dermoscopy images. Biomed Signal Process Control 6(4):395-404

51. Abbas Q, Garcia IF, Emre Celebi M, Ahmad W (2013) A feature-preserving hair removal algorithm for dermoscopy images. Skin Res Technol 19(1):e27-e36

52. Toossi MTB, Pourreza HR, Zare H, Sigari MH, Layegh P, Azimi A (2013) An effective hair removal algorithm for dermoscopy images. Skin Res Technol 19(3):230-235

53. Mirzaalian H, Lee TK, Hamarneh G (2014) Hair enhancement in dermoscopic images using dual-channel quaternion tubularness filters and MRF-based multilabel optimization. IEEE Trans Image Process 23(12):5486-5496

54. Lee T, Ng V, Gallagher R, Coldman A, McLean D (1997) Dullrazor $^{\circledR}$ : a software approach to hair removal from images. Comput Biol Med 27(6):533-543

55. Celebi ME, Iyatomi H, Schaefer G, Stoecker WV (2009) Lesion border detection in dermoscopy images. Comput Med Imaging Graph 33(2):148-153

56. Celebi ME, Wen Q, Iyatomi H, Shimizu K, Zhou H, Schaefer G (2015) A State-of-the-art survey on lesion border detection in dermoscopy images. In: Celebi ME, Mendonca T, Marques JS (eds) Dermoscopy image analysis. CRC Press, Boca Raton, pp 97-129

57. Fukunaga K (1990) Introduction to statistical pattern recognition, 2nd edn. Academic Press, San Diego

58. Webb AR (2003) Statistical pattern recognition, 2nd edn. Wiley, England

59. Guyon I, Gunn S, Nikravesh M, Zadeh L (2006) Feature extraction: foundations and applications, vol 207. Studies in fuzziness and soft computing. Springer, Berlin

60. Liu H, Motoda H (1998) Feature extraction, construction and selection: a data mining perspective. Springer, Norwell

61. Ma L, Staunton RC (2013) Analysis of the contour structural irregularity of skin lesions using wavelet decomposition. Pattern Recognit 46(1):98-106

62. Wighton P, Lee TK, Lui H, McLean D, Atkins MS (2011) Generalizing common tasks in automated skin lesion diagnosis. IEEE Trans Inf Technol Biomed 15(4):622-629

63. Maglogiannis I, Delibasis KK (2015) Enhancing classification accuracy utilizing globules and dots features in digital dermoscopy. Comput Methods Programs Biomed 118(2):124-133

64. Shrestha B, Bishop J, Kam K, Chen X, Moss RH, Stoecker WV, Umbaugh S, Stanley RJ, Celebi ME, Marghoob AA (2010) Detection of atypical texture features in early malignant melanoma. Skin Res Technol 16(1):60-65

65. Abbasi NR, Shaw HM, Rigel DS, Friedman RJ, McCarthy WH, Osman I, Kopf AW, Polsky D (2004) Early diagnosis of cutaneous melanoma: revisiting the ABCD criteria. JAMA 292(22):2771-2776

66. Blum A, Rassner G, Garbe C (2003) Modified ABC-point list of dermoscopy: a simplified and highly accurate dermoscopic algorithm for the diagnosis of cutaneous melanocytic lesions. J Am Acad Dermatol 48(5):672-678

67. Johr RH (2002) Dermoscopy: alternative melanocytic algorithms - the ABCD rule of dermatoscopy, menzies scoring method, and 7-point checklist. Clin Dermatol 20(3):240-247

68. Braun RP, Rabinovitz HS, Oliviero M, Kopf AW, Saurat J-H (2005) Dermoscopy of pigmented skin lesions. J Am Acad Dermatol 52(1):109-121
69. Argenziano G, Fabbrocini G, Carli P, De Giorgi V, Sammarco E, Delfino M (1998) Epiluminescence microscopy for the diagnosis of doubtful melanocytic skin lesions: comparison of the abcd rule of dermatoscopy and a new 7-point checklist based on pattern analysis. Arch Dermatol 134(12):1563-1570

70. Cavalcanti PG, Scharcanski J (2011) Automated prescreening of pigmented skin lesions using standard cameras. Comput Med Imaging Graph 35(6):481-491

71. Situ N, Yuan X, Zouridakis G (2011) Assisting main task learning by heterogeneous auxiliary tasks with applications to skin cancer screening. J Mach Learn Res 15:688-697

72. Sadeghi M, Lee TK, McLean D, Lui H, Atkins MS (2012) Global pattern analysis and classification of dermoscopic images using textons. In: SPIE, vol 8314. Medical imaging 2012: image processing, San Diego, February 4-9. International Society for Optics and Photonics, pp 83144X-83146

73. Abbas Q, Celebi ME, Serrano C, Fondón García I, Ma G (2013) Pattern classification of dermoscopy images: a perceptually uniform model. Pattern Recognit 46(1):86-97

74. Isasi AG, Zapirain BG, Zorrilla AM (2011) Melanomas noninvasive diagnosis application based on the ABCD rule and pattern recognition image processing algorithms. Comput Biol Med 41(9):742-755

75. Argenziano G, Soyer HP, Chimenti S, Talamini R, Corona R, Sera F, Binder M, Cerroni L, De Rosa G, Ferrara G, Hofmann-Wellenhof R, Landthaler M, Menzies SW, Pehamberger H, Piccolo D, Rabinovitz HS, Schiffner R, Staibano S, Stolz W, Bartenjev I, Blum A, Braun R, Cabo H, Carli P, De Giorgi V, Fleming MG, Grichnik JM, Grin CM, Halpern AC, Johr R, Katz B, Kenet RO, Kittler H, Kreusch J, Malvehy J, Mazzocchetti G, Oliviero M, Özdemir F, Peris K, Perotti R, Perusquia A, Pizzichetta MA, Puig S, Rao B, Rubegni P, Saida T, Scalvenzi M, Seidenari S, Stanganelli I, Tanaka M, Westerhoff K, Wolf IH, Braun-Falco O, Kerl H, Nishikawa T, Wolff K, Kopf AW (2003) Dermoscopy of pigmented skin lesions: results of a consensus meeting via the internet. J Am Acad Dermatol 48(5):679-693

76. Argenziano G, Soyer H, De Giorgi V, Piccolo D, Carli P, Delfino $M$ et al (2002) Dermoscopy: a tutorial. EDRA Medical Publishing \& New Media, Milan, Italy

77. Celebi ME, Iyatomi H, Stoecker WV, Moss RH, Rabinovitz HS, Argenziano G, Soyer HP (2008) Automatic detection of bluewhite veil and related structures in dermoscopy images. Comput Med Imaging Graph 32(8):670-677

78. Betta G, Di Leo G, Fabbrocini G, Paolillo A, Scalvenzi M (2005) Automated application of the "7-point checklist" diagnosis method for skin lesions: estimation of chromatic and shape parameters. In: Instrumentation and measurement technology conference, Ottawa, May 16-19. IEEE, pp 1818-1822

79. Leo GD, Fabbrocini G, Paolillo A, Rescigno O, Sommella P (2009) Towards an automatic diagnosis system for skin lesions: estimation of blue-whitish veil and regression structures. In: International multi-conference on systems, signals and devices, Djerba, March 23-26. IEEE, pp 1-6

80. Zortea M, Schopf TR, Thon K, Geilhufe M, Hindberg K, Kirchesch H, Møllersen K, Schulz J, Skrøvseth SO, Godtliebsen F (2014) Performance of a dermoscopy-based computer vision system for the diagnosis of pigmented skin lesions compared with visual evaluation by experienced dermatologists. Artif Intell Med 60(1):13-26

81. Silva CS, Marcal AR (2013) Colour-based dermoscopy classification of cutaneous lesions: an alternative approach. Comput Methods Biomech Biomed Eng Imaging Vis 1(4):211-224

82. Giotis I, Molders N, Land S, Biehl M, Jonkman MF, Petkov N (2015) MED-NODE: a computer-assisted melanoma diagnosis system using non-dermoscopic images. Expert Syst Appl 42(19):6578-6585 
83. Barata C, Emre Celebi M, Marques JS (2015) Melanoma detection algorithm based on feature fusion. In: 37th annual international conference of the IEEE Engineering in Medicine and Biology Society, Milan, August 25-29. IEEE, pp 2653-2656

84. Rastgoo M, Garcia R, Morel O, Marzani F (2015) Automatic differentiation of melanoma from dysplastic nevi. Comput Med Imaging Graph 43:44-52

85. Barata C, Marques JS, Celebi ME (2013) Towards an automatic bag-of-features model for the classification of dermoscopy images: the influence of segmentation. In: Proceedings of the 8th international symposium on image and signal processing and analysis, Trieste, September 4-6. IEEE, pp 274-279

86. Sáez A, Acha B, Serrano C (2014) Pattern analysis in dermoscopic images. In: Scharcanski J, Celebi ME (eds) Computer vision techniques for the diagnosis of skin cancer. Series in BioEngineering. Springer, Berlin, pp 23-48

87. Chang Y, Stanley RJ, Moss RH, Van Stoecker W (2005) A systematic heuristic approach for feature selection for melanoma discrimination using clinical images. Skin Res Technol 11(3):165-178

88. Celebi ME, Kingravi HA, Uddin B, Iyatomi H, Aslandogan YA, Stoecker WV, Moss RH (2007) A methodological approach to the classification of dermoscopy images. Comput Med Imaging Graph 31(6):362-373

89. Ng VTY, Fung BYM, Lee TK (2005) Determining the asymmetry of skin lesion with fuzzy borders. Comput Biol Med 35(2): 103-120

90. Oliveira RB, Marranghello N, Pereira AS, Tavares JMRS (2016) A computational approach for detecting pigmented skin lesions in macroscopic images. Expert Syst Appl 61:53-63

91. D’Amico M, Ferri M, Stanganelli I (2004) Qualitative asymmetry measure for melanoma detection. In: Proceedings of the 2004 IEEE international symposium on biomedical imaging: nano-macro, Arlington, April 15-18. IEEE, pp 1155-1158

92. Lee TK, McLean DI, Atkins MS (2003) Irregularity index: a new border irregularity measure for cutaneous melanocytic lesions. Med Image Anal 7(1):47-64

93. Iyatomi H, Norton K, Celebi ME, Schaefer G, Tanaka M, Ogawa K (2010) Classification of melanocytic skin lesions from non-melanocytic lesions. In: Annual international conference of the IEEE Engineering in Medicine and Biology Society, Buenos Aires, August 31-September 4. IEEE, pp 5407-5410

94. Iyatomi H, Oka H, Celebi ME, Hashimoto M, Hagiwara M, Tanaka M, Ogawa K (2008) An improved Internet-based melanoma screening system with dermatologist-like tumor area extraction algorithm. Comput Med Imaging Graph 32(7):566-579

95. Jaworek-Korjakowska J (2015) Novel method for border irregularity assessment in dermoscopic color images. Comput Math Methods Med 2015:1-11 (article ID 496202)

96. Iyatomi H, Oka H, Celebi ME, Ogawa K, Argenziano G, Soyer HP, Koga H, Saida T, Ohara K, Tanaka M (2008) Computerbased classification of dermoscopy images of melanocytic lesions on acral volar skin. J Investig Dermatol 128(8):2049-2054

97. Dalal A, Moss RH, Stanley RJ, Stoecker WV, Gupta K, Calcara DA, Xu J, Shrestha B, Drugge R, Malters JM, Perry LA (2011) Concentric decile segmentation of white and hypopigmented areas in dermoscopy images of skin lesions allows discrimination of malignant melanoma. Comput Med Imaging Graph 35(2):148-154

98. Mendoza CS, Serrano C, Acha B (2009) Scale invariant descriptors in pattern analysis of melanocytic lesions. In: 16th IEEE international conference on image processing, Cairo, November 7-10. IEEE, pp 4193-4196
99. Khan A, Gupta K, Stanley RJ, Stoecker WV, Moss RH, Argenziano G, Soyer HP, Rabinovitz HS, Cognetta AB (2009) Fuzzy logic techniques for blotch feature evaluation in dermoscopy images. Comput Med Imaging Graph 33(1):50-57

100. Barata C, Marques JS, Rozeira J (2012) A system for the detection of pigment network in dermoscopy images using directional filters. IEEE Trans Biomed Eng 59(10):2744-2754

101. Sadeghi M, Razmara M, Wighton P, Lee TK, Atkins MS (2010) Modeling the dermoscopic structure pigment network using a clinically inspired feature set. In: Dohi T, Sakuma I, Liao H (eds) Medical imaging and augmented reality. Springer, Berlin, pp 467-474

102. Møllersen K, Zortea M, Hindberg K, Schopf TR, Skrøvseth SO, Godtliebsen F (2015) Improved skin lesion diagnostics for general practice by computer-aided diagnostics. In: Celebi ME, Mendonca T, Marques JS (eds) Dermoscopy image analysis. CRC Press, Boca Raton, pp 247-292

103. Abbas Q, Celebi ME, Garcia IF, Ahmad W (2013) Melanoma recognition framework based on expert definition of $\mathrm{ABCD}$ for dermoscopic images. Skin Res Technol 19(1):e93-e102

104. Zhou Y, Smith M, Smith L, Warr R (2010) A new method describing border irregularity of pigmented lesions. Skin Res Technol 16(1):66-76

105. Shimizu K, Iyatomi H, Celebi ME, Norton K-A, Tanaka M (2015) Four-class classification of skin lesions with task decomposition strategy. IEEE Trans Biomed Eng 62(1):274-283

106. Clawson KM, Morrow P, Scotney B, McKenna J, Dolan O (2009) Analysis of pigmented skin lesion border irregularity using the harmonic wavelet transform. In: 13th international machine vision and image processing conference, Dublin, September 2-4. IEEE, pp 18-23

107. Schmid-Saugeon P (2000) Symmetry axis computation for almost-symmetrical and asymmetrical objects: application to pigmented skin lesions. Med Image Anal 4(3):269-282

108. Maglogiannis I, Doukas CN (2009) Overview of advanced computer vision systems for skin lesions characterization. IEEE Trans Inf Technol Biomed 13(5):721-733

109. Rahman MM, Bhattacharya P, Desai BC (2008) A multiple expert-based melanoma recognition system for dermoscopic images of pigmented skin lesions. In: International conference on bioinformatics and bioengineering, Athens, October 8-10. IEEE, pp 1-6

110. Schaefer G, Krawczyk B, Celebi ME, Iyatomi H (2014) An ensemble classification approach for melanoma diagnosis. Memet Comput 6(4):233-240

111. Arroyo JLG, Zapirain BG (2014) Detection of pigment network in dermoscopy images using supervised machine learning and structural analysis. Comput Biol Med 44:144-157

112. Abedini M, Chen Q, Codella NCF, Garnavi R, Sun X (2015) Accurate and scalable system for automatic detection of malignant melanoma. In: Celebi ME, Mendonca T, Marques JS (eds) Dermoscopy image analysis. CRC Press, Boca Raton, pp 293-343

113. Iyatomi H, Celebi ME, Schaefer G, Tanaka M (2011) Automated color calibration method for dermoscopy images. Comput Med Imaging Graph 35(2):89-98

114. Celebi ME, Zornberg A (2014) Automated quantification of clinically significant colors in dermoscopy images and its application to skin lesion classification. IEEE Syst $\mathbf{J}$ 8(3):980-984

115. Barata C, Figueiredo MA, Celebi ME, Marques JS (2014) Color identification in dermoscopy images using gaussian mixture models. In: Proceedings of the IEEE international conference on acoustics, speech and signal processing, Florence, May 4-9. IEEE, pp 3611-3615 
116. Barata C, Celebi ME, Marques JS Color (2015) Detection in dermoscopy images based on scarce annotations. In: 7th Iberian conference on pattern recognition and image analysis, Santiago de Compostela, June 17-19. Springer, pp 309-316

117. Stoecker WV, Gupta K, Stanley RJ, Moss RH, Shrestha B (2005) Detection of asymmetric blotches (asymmetric structureless areas) in dermoscopy images of malignant melanoma using relative color. Skin Res Technol 11(3):179-184

118. Madooei A, Drew MS (2013) A colour palette for automatic detection of blue-white veil. In: Proceedings of the 21st color and imaging conference final program and proceedings, Albuquerque, November 4-8. Society for Imaging Science and Technology, pp 200-205

119. Madooei A, Drew MS, Sadeghi M, Atkins MS (2013) Automatic detection of blue-white veil by discrete colour matching in dermoscopy images. In: Proceedings of the 16th international conference on medical image computing and computer-assisted intervention, Nagoya, September 22-26. Springer, Berlin, pp 453-460

120. Madasu VK, Lovell BC (2009) Blotch detection in pigmented skin lesions using fuzzy co-clustering and texture segmentation. In: Digital image computing: techniques and applications, Melbourne, December 1-3. IEEE, pp 25-31

121. Tanaka T, Torii S, Kabuta I, Shimizu K, Tanaka M (2008) Pattern classification of nevus with texture analysis. IEEJ Trans Electr Electron Eng 3(1):143-150

122. Anantha M, Moss RH, Stoecker WV (2004) Detection of pigment network in dermatoscopy images using texture analysis. Comput Med Imaging Graph 28(5):225-234

123. Yuan X, Yang Z, Zouridakis G, Mullani N (2006) SVM-based texture classification and application to early melanoma detection. In: 28th annual international conference of the IEEE Engineering in Medicine and Biology Society, New York, August 30-September 3. IEEE, pp 4775-4778

124. Serrano C, Acha B (2009) Pattern analysis of dermoscopic images based on Markov random fields. Pattern Recognit 42(6):1052-1057

125. Amelard R, Glaister J, Wong A, Clausi DA (2015) High-level intuitive features (HLIFs) for intuitive skin lesion description. IEEE Trans Biomed Eng 62(3):820-831

126. Torre EL, Caputo B, Tommasi T (2010) Learning methods for melanoma recognition. Int $\mathrm{J}$ Imaging Syst Technol 20(4):316-322

127. Huang H, Bergstresser P (2007) A new hybrid technique for dermatological image registration. In: 7th IEEE international conference on bioinformatics and bioengineering, Boston, October 14-17. IEEE, pp 1163-1167

128. Skrovseth SO, Schopf TR, Thon K, Zortea M, Geilhufe M, Mollersen K, Kirchesch HM, Godtliebsen F (2010) A computer aided diagnostic system for malignant melanomas. In: 3rd international symposium on applied sciences in biomedical and communication technologies, Rome, November 7-10. IEEE, pp 1-5

129. Jaworek-Korjakowska J, Tadeusiewicz R (2014) Determination of border irregularity in dermoscopic color images of pigmented skin lesions. In: 36th annual international conference of the IEEE Engineering in Medicine and Biology Society, Chicago, August 26-30. IEEE, pp 6459-6462

130. Xie X (2008) A review of recent advances in surface defect detection using texture analysis techniques. Electron Lett Comput Vis Image Anal 7(3):1-22

131. Haralick RM, Shanmugam K, Dinstein IH (1973) Textural features for image classification. IEEE Trans Syst Man Cybern 6:610-621

132. Al-Akaidi M (2004) Fractal speech processing. Cambridge University Press, New York
133. Strayer SM, Reynolds P (2003) Diagnosing skin malignancy: assessment of predictive clinical criteria and risk factors. J Fam Pract 52(3):210-218

134. Hani AFM, Fitriyah H, Prakasa E, Asirvadam VS, Hussein SH, Azura MA (2010) In vivo 3D thickness measurement of skin lesion. In: IEEE conference on biomedical engineering and sciences, Kuala Lumpur, November 30-December 2. IEEE, pp $155-160$

135. Fadzil MA, Fitriyah H, Prakasa E, Nugroho H, Hussein SH, Affandi AM (2009) Thickness characterization of 3D skin surface images using reference line construction approach. In: International visual informatics conference, Kuala Lumpur, November 11-13. Springer, Berlin, pp 448-454

136. Mirzaalian H, Lee TK, Hamarneh G (2016) Skin lesion tracking using structured graphical models. Med Image Anal 27:84-92

137. Guyon I, Elisseeff A (2003) An introduction to variable and feature selection. J Mach Learn Res 3:1157-1182

138. Han J, Kamber M (2006) Data mining: concepts and techniques. Elsevier, San Francisco

139. Dash M, Liu H (1997) Feature selection for classification. Intell Data Anal 1(3):131-156

140. Witten IH, Frank E, Hall MA (2011) Data mining: practical machine learning tools and techniques. Morgan Kaufmann, San Francisco

141. Liu H, Yu L (2005) Toward integrating feature selection algorithms for classification and clustering. IEEE Trans Knowl Data Eng 17(4):491-502

142. Kohavi R, John GH (1997) Wrappers for feature subset selection. Artif Intell 97(1):273-324

143. Hand D, Mannila H, Smyth P (2001) Principles of data mining. The MIT Press, London

144. Chawla NV (2005) Data mining for imbalanced datasets: an overview. In: Maimon O, Rokach L (eds) Data mining and knowledge discovery handbook. Springer, New York, pp 853-867

145. Chawla NV, Bowyer KW, Hall LO, Kegelmeyer WP (2002) SMOTE: synthetic minority over-sampling technique. J Artif Intell Res 16:321-357

146. Congdon P (2007) Bayesian statistical modelling, vol 704, 2nd edn. Wiley, Chichester

147. Haykin SS (1999) Neural networks: a comprehensive foundation. Prentice Hall, Englewood Cliffs

148. Burges CJC (1998) A tutorial on support vector machines for pattern recognition. Data Min Knowl Discov 2(2):121-167

149. Cavalcanti PG, Scharcanski J, Baranoski GV (2013) A twostage approach for discriminating melanocytic skin lesions using standard cameras. Expert Syst Appl 40(10):4054-4064

150. Arroyo JLG, Zapirain BG, Zorrilla AM (2011) Blue-white veil and dark-red patch of pigment pattern recognition in dermoscopic images using machine-learning techniques. In: IEEE international symposium on signal processing and information technology, Bilbao, December 14-17. IEEE, pp 196-201

151. Fabbrocini G, Betta G, Di Leo G, Liguori C, Paolillo A, Pietrosanto A, Sommella P, Rescigno O, Cacciapuoti S, Pastore F (2010) Epiluminescence image processing for melanocytic skin lesion diagnosis based on 7-point check-list: a preliminary discussion on three parameters. Open Dermatol J 4:110-115

152. Mirzaalian H, Lee TK, Hamarneh G (2012) Learning features for streak detection in dermoscopic color images using localized radial flux of principal intensity curvature. In: Workshop on mathematical methods in biomedical image analysis, Breckenridge, January 9-10. IEEE, pp 97-101

153. Schaefer G, Krawczyk B, Celebi ME, Iyatomi H, Hassanien AE (2014) Melanoma classification based on ensemble classification of dermoscopy image features. In: International conference on advanced machine learning technologies and applications, Cairo, November 28-30. Springer, pp 291-298 
154. Sadeghi M, Lee TK, McLean D, Lui H, Atkins MS (2013) Detection and analysis of irregular streaks in dermoscopic images of skin lesions. IEEE Trans Med Imaging 32(5):849-861

155. Fleming MG, Steger C, Zhang J, Gao J, Cognetta AB, Dyer CR (1998) Techniques for a structural analysis of dermatoscopic imagery. Comput Med Imaging Graph 22(5):375-389

156. Abedini M, Codella NCF, Connell JH, Garnavi R, Merler M, Pankanti S, Smith JR, Syeda-Mahmood T (2015) A generalized framework for medical image classification and recognition. IBM J Res Dev 59(2/3):1

157. Dietterich TG (2000) Ensemble methods in machine learning. In: International workshop on multiple classifier systems, Italy, June 21-23. Springer, Berlin, pp 1-15

158. Breiman L (2001) Random forests. Mach Learn 45(1):5-32

159. Zhu J, Zou H, Rosset S, Hastie T (2009) Multi-class adaboost. Stat Interface 2(3):349-360

160. Fawcett T (2004) ROC graphs: notes and practical considerations for researchers. Mach Learn 31(1):1-38

161. Celebi ME, Kingravi HA, Iyatomi H, Alp Aslandogan Y, Stoecker WV, Moss RH, Malters JM, Grichnik JM, Marghoob AA, Rabinovitz HS, Menzies SW (2008) Border detection in dermoscopy images using statistical region merging. Skin Res Technol 14(3):347-353

162. Abbas Q, Celebi ME, Fondón I (2012) Computer-aided pattern classification system for dermoscopy images. Skin Res Technol 18(3):278-289
163. Barhoumi W, Baâzaoui A (2014) Pigment network detection in dermatoscopic images for melanoma diagnosis. IRBM 35(3): 128-138

164. Leo GD, Liguori C, Paolillo A, Sommella P (2008) An improved procedure for the automatic detection of dermoscopic structures in digital ELM images of skin lesions. In: IEEE conference on virtual environments, human-computer interfaces and measurement systems, Istanbul, July 14-16. IEEE, pp 190-194

165. Grana C, Cucchiara R, Pellacani G, Seidenari S (2006) Line detection and texture characterization of network patterns. In: 18th international conference on pattern recognition, Hong Kong, August 20-24. IEEE, pp 275-278

166. Betta G, Di Leo G, Fabbrocini G, Paolillo A, Sommella P (2006) Dermoscopic image-analysis system: estimation of atypical pigment network and atypical vascular pattern. In: IEEE international workshop on medical measurement and applications, Benevento, April 20-21. IEEE, pp 63-67

167. Celebi ME, Schaefer G (2012) Color medical image analysis, vol 6. Springer, Dordrecht

168. Celebi ME, Mendonca T, Marques JS (2015) Dermoscopy Image analysis, vol 10. CRC Press, Boca Raton

169. Mayer J (1997) Systematic review of the diagnostic accuracy of dermatoscopy in detecting malignant melanoma. Med J Aust 167(4):206-210 